\title{
Management of radiotherapy patients with implanted cardiac pacemakers and defibrillators: A Report of the AAPM TG-203 ${ }^{\dagger}$
}

Moyed Miften ${ }^{\mathrm{a}),}$

Task Group 203, Department of Radiation Oncology, University of Colorado School of Medicine, Aurora, CO 80045, USA

Dimitris Mihailidis ${ }^{a), \$}$

Task Group 203, University of Pennsylvania Perelman Center for Advanced Medicine, Philadelphia, PA 19104, USA

Stephen F. Kry

Department of Radiation Physics, UT MD Anderson Cancer Center, Houston, TX 77030, USA

Chester Reft

Department of Radiation Oncology, University of Chicago, Chicago, IL 60637, USA

Carlos Esquivel

Department of Radiation Oncology, UT Health Sciences Center, San Antonio, TX 78229, USA

Jonathan Farr

Division of Radiological Sciences, St. Jude Children's Research Hospital, Memphis, TN 38105, USA

David Followill

Department of Radiation Physics, UT MD Anderson Cancer Center, Houston, TX 77030, USA

Coen Hurkmans

Department of Radiotherapy, Catharina Hospital, Eindhoven, the Netherlands

Arthur Liu

Department of Radiation Oncology, University of Colorado School of Medicine, Aurora, CO 80045, USA

Olivier Gayou

Department of Radiation Oncology, Allegheny General Hospital, Pittsburg, PA 15212, USA

Michael Gossman

Department of Radiation Oncology, Tri-State Regional Cancer Center, Ashland, KY 41101, USA

Mahadevappa Mahesh

Department of Radiology, Johns Hopkins University School of Medicine, Baltimore, MD 21287, USA

Richard Popple

Department of Radiation Oncology, University of Alabama, Birmingham, AL 35249, USA

Joann Prisciandaro

Department of Radiation Oncology, University of Michigan, Ann Arbor, MI 48109, USA

Jeffrey Wilkinson

Consultant, Medtronic Inc., Mounds View, MN55112, USA

(Received 4 April 2019; revised 16 July 2019; accepted for publication 28 August 2019; published 1 November 2019)

Managing radiotherapy patients with implanted cardiac devices (implantable cardiac pacemakers and implantable cardioverter-defibrillators) has been a great practical and procedural challenge in radiation oncology practice. Since the publication of the AAPM TG-34 in 1994, large bodies of literature and case reports have been published about different kinds of radiation effects on modern technology implantable cardiac devices and patient management before, during, and after radiotherapy. This task group report provides the framework that analyzes the potential failure modes of these devices and lays out the methodology for patient management in a comprehensive and concise way, in every step of the entire radiotherapy process. (C) 2019 American Association of Physicists in Medicine [https:// doi.org/10.1002/mp.13838]

Key words: cardiac implantable electronic devices (CIED), device malfunction, implantable cardiac pacemakers (ICP), implantable cardioverter-defibrillators (ICD), patient management, radiation damage 


\section{TABLE OF CONTENTS}

\begin{tabular}{|c|}
\hline \multirow{2}{*}{$\begin{array}{l}\text { 1. Purpose } \\
\text { 2. Introduction }\end{array}$} \\
\hline \\
\hline 3. ICPs AND ICD Devices \\
\hline 3.A. Design and function \\
\hline 3.B. Sensitivity and potential failures \\
\hline 4. Conditions in clinical use that can induce malfunctions \\
\hline 4.A. Cumulative dose effects \\
\hline 4.B. Neutron-induced upsets \\
\hline 4.C. Dose-rate effects \\
\hline 4.D. Magnetic fields \\
\hline 4.E. Electromagnetic Interference (EMI) \\
\hline 5. Malfunction risk associated with clinical procedures \\
\hline 5.A. Imaging \\
\hline 5.A.1. $\mathrm{kV}$ Imaging for treatment planning and localization \\
\hline 5.A.2. MV imaging for treatment localization \\
\hline 5.A.3. MR imaging \\
\hline 5.B. Therapy \\
\hline 5.B.1. $k V$ therapy treatment units \\
\hline 5.B.2. Brachytherapy \\
\hline 5.B.3. MV photon and electron beams \\
\hline 5.B.4. Neutron and proton beams \\
\hline 6. Dose assessment considerations \\
\hline 6.A. Where to assess dose \\
\hline 6.B. Medium specification \\
\hline 6.C. Dose assessment \\
\hline 6.C.1. Calculation \\
\hline 6.C.2. Measurement \\
\hline 6.C.3. Typical out-of-field doses \\
\hline 7. Recommended dose thresholds \\
\hline 8. Patient management and recommendations \\
\hline 8.A. Staff and department requirements \\
\hline 8.B. Measures before treatment \\
\hline 8.C. Simulation \\
\hline 8.D. Treatment planning \\
\hline 8.E. Measures during treatment \\
\hline 8.F. Documentation and record keeping \\
\hline 9. Management of patients with other implantable devices \\
\hline 10. Summary and conclusions \\
\hline
\end{tabular}

\section{PURPOSE}

The objective of this report was to review published literature evaluate all possible effects of therapeutic radiations on cardiac implantable electronic devices (CIEDs), provide methods to clinically assess the dose levels, provide an estimate of the risks for the various radiation modalities associate lethal and nonlethal types of device failures, recommend methods to manage patients with such devices, as well as to develop recommendations in order to minimize the damage to the devices during radiotherapy (RT) procedures.

\section{INTRODUCTION}

The American Association of Physicists in Medicine (AAPM) Task Group 34 (TG-34) provided a set of guidelines on the management of cancer patients with implanted cardiac pacemakers receiving $\mathrm{RT}^{1}$. The report provided valuable information on the effects of radiation on implantable pacemakers and suggested a protocol to manage such patients. One of the main recommendations of TG-34 was that treatment planning results should yield no more than 2 Gy to any part of the pacemaker. While TG-34 presented valuable recommendations when the report was published in 1994, the recommendations then dealt with pacemakers only and were based on older device technology, and older delivery methods.

In a contemporary setting, management is further complicated as it is common to encounter implantable cardiac pacemakers (ICPs) as well as implantable cardioverterdefibrillators (ICDs), collectively referred to as CIEDs, to indicate that they require a source of electrical power for their function. For these modern devices, numerous investigations $^{2-14}$ have dealt with their functionality during RT treatments, including the effects of dose, dose-rate, and secondary neutrons to these devices from conformal RT, intensity-modulated RT, and proton beam therapy. Some research has even dealt with the effects of imaging procedures on ICPs and ICDs. ${ }^{14-16}$ However, in spite of the availability of TG-34 and other published research, ${ }^{2-16}$ major discrepancies still exist among manufacturer recommendations and clinic policies regarding patient management and safety guidance. ${ }^{5}$ Furthermore, some of this information, particularly on malfunction limits and mechanisms, is somewhat confusing and often contradictory. For example, some devices have suffered deleterious effects ${ }^{4}$ at a dose of $0.15 \mathrm{~Gy}$ at a dose-rate of only $0.2 \mathrm{~Gy} / \mathrm{min}$, while others exhibited dose tolerance above $20 \mathrm{~Gy}$. There has been a reported pacemaker software failure at 0.11 Gy of scattered dose, which the authors attributed to electromagnetic interference (EMI) effects during RT. ${ }^{17}$ Although it should be noted that it is impossible to isolate EMI effects from other radiation effects, so many such errors have unknown origin. Mouton et al., ${ }^{4}$ reported changes in CIED output for an $18 \mathrm{MV}$ beam at total doses of as little as $0.15 \mathrm{~Gy}$, concluding changes are more likely due to a singleevent upset (SEU) induced by photoneutrons ${ }^{10,18}$ rather than from a high dose-rate. Assessment of malfunctions in these devices, and corresponding management in clinical situations, are clearly complicated by malfunctions attributed to a range of possible mechanisms.

The challenges posed by the lack of consistent information were made clear by the work of Solan et al., ${ }^{5}$ who, in 2004, published a survey of clinical practice patterns among 75 radiation oncology departments in the United States and Canada for management of CIED patients during RT. It was found that only $31 \%$ of radiation facilities limit the total allowable dose exposure to the device, whereas $20 \%$ of facilities strictly follow TG-34 guidelines; only 15\% have a management policy; $37 \%$ of the clinics consult a cardiologist; and $33 \%$ contact the CIEDs manufacturer for advice. A mere $35 \%$ monitor the patient during treatment and only $20 \%$ of the clinics perform in vivo dose measurements regularly. Similar inconsistent or insufficient practices were observed by Lester et al. ${ }^{19}$ based on a survey across the United Kingdom, and by Soejima et al., ${ }^{20}$ 
in a survey of centers in Japan. Gossman et al., ${ }^{21}$ in a retrospective physicians' survey on treatment approach, delivery and follow-up for management of RT patients with CIEDs, concluded that most radiation oncologists were unfamiliar with the recommended standard of practice by the AAPM, and the involvement of the medical physicist (MP) with patient management, finding both to be insufficient. The absence of, and need for, clearly defined and up-to-date set of guidelines with a uniform approach to the management of these patients is apparent.

Even after 20 or so years following the publication of TG34 , not all centers adhere to its recommendations. As shown by Solan et al., in $2004^{5}$ and by Soejima et al., ${ }^{20}$ radiation oncologists failed to communicate radiation safety concerns with implanting physicians, even though in most instances those concerns have resulted in device explantation. Education and scientific awareness appears to be markedly unexploited.

In 2017, the Heart Rhythm Society (HRS) published their expert consensus statement on cardiac devices. While the focus of this report was on MRI interactions with CIEDs, this report also discussed management of RT patients (Section 7 in that report). ${ }^{22}$ In their report, HRS focused on avoiding the use of neutron-producing beams (e.g., photons of $\mathrm{E}>10 \mathrm{MV}$ or protons), and considered a 5 Gy dose threshold, but were ultimately unable to make a recommendation concerning cumulative dose risks. While the HRS report provides some framework for patient management, it does not offer detailed recommendations on the management of these patients, nor does it provide guidance on dose assessment to CIEDs, both of which are key components of the current task group report. In addition, HRS guidelines did not provide clear recommendations on several issues, including how to manage the patient if there is a discrepancy between a 5 Gy society-based dose threshold and manufacturer's recommended dose threshold. Therefore, while guidelines are typically consistent between this task group report and the HRS report (a notable exception being the inclusion of 2 and 5 Gy cumulative dose thresholds for CIEDs), there are substantial differences in the focus of the reports.

It is the intent of TG-203 to provide more up-to-date formal guidance as well as an initial mechanism for wider communication between professionals in the radiotherapy community and in the field of cardiac rhythm management. Furthermore, the complementary information of the 2017 HRS expert consensus and TG-203 will be beneficial for the users of new MRI-linac RT technologies. ${ }^{23}$ Finally, it should be noted that the successful treatment of patients implanted with CIEDs requires a cooperative effort involving not only the expertise of the MP but also the close involvement of the radiation oncologist, cardiologist, radiation therapist, nurse, and electrophysiologist.

\section{ICP AND ICD DEVICES}

\section{A. Design and function}

Implantable cardiac pacemakers are devices designed to maintain cardiac rhythm and are usually implanted subcutaneously in the chest or in the abdomen. In general, the device is positioned overlying the pectoral muscles, although occasionally implanted in the abdominal wall. ICPs sense continuously and pace when intervals exceed the programmed limits. During pacing, they emit electrical pulses to prompt the normal rate while during sensing they monitor the heart's natural activity. ICDs function similar to ICPs except that they use a capacitor to deliver a high-voltage shock to the heart when needed. ICPs are generally prescribed for the prevention of bradycardia, cardiac arrest, and more recently in the treatment of congestive heart disease. ICDs are implanted to treat symptomatic ventricular tachycardia (VT) and fibrillation or to prevent sudden cardiac death in highrisk cardiac patients. Both devices consist of a battery-powered pulse generator with encased electronics that is connected to pacing leads that serve both to monitor the cardiac functions and to deliver treatment (Fig. 1). The primary manufacturers of CIEDs in the United States are Medtronic, Inc. (Minneapolis, MN, USA), Boston Scientific (Boston, MA, USA), and St. Jude Medical (St. Paul, MN, USA). A list of major device manufacturers is presented in Table I.

Both ICPs and ICDs have undergone substantial improvements in function since the publication of TG-34 in1994, with more complex therapies, better diagnostics, an increase in the number of the heart's chambers that are sensed and controlled, smaller sizes, and reduced power consumption. Although the concurrent implant of pacers and defibrillators was common in the early 1990s, this practice has been largely abandoned due to the increased sophistication of the implantable defibrillator.

Modern CIEDs utilize complementary metal oxide semiconductor (CMOS) technology in very-large-scale integrated circuits to provide these increased capabilities. The advantages of CMOS technology are high noise immunity, greater reliability, and lower power consumption. However, these advantages might come at the cost of higher susceptibility to radiation effects. The CMOS technology available in the early 1990s was generally more susceptible to permanent damage from ionizing radiation than the previously encountered bipolar transistor technology. ${ }^{1,24}$ Several cases have been reported of their failure both in vivo ${ }^{25-29}$ and in vitro. ${ }^{30-34}$ Modern devices require as many as 50,000,000 CMOS transistors compared to less than 1,000 in the most advanced 1994 bipolar designs. This increased circuit complexity and ultra-lowpower consumption are largely responsible for the continued sensitivity to ionizing radiation, rather than the integrated circuit technology itself.

The ICP produces a small electrical stimulus that is sufficient to begin a heartbeat by depolarizing the small portion of the myocardium immediately adjacent to the pacing electrode. The RF transducer provides an RF signal for interrogating the device. The ICP is most often adjusted to sense the heart's intrinsic rhythm and only discharges pulses when a beat is missed, referred to as "demand pacing". Pacing electrodes may be implanted to sense or control the right atrium, right ventricle, or left ventricle depending on the patient's disease status and lifestyle requirements. The ICP is either a 


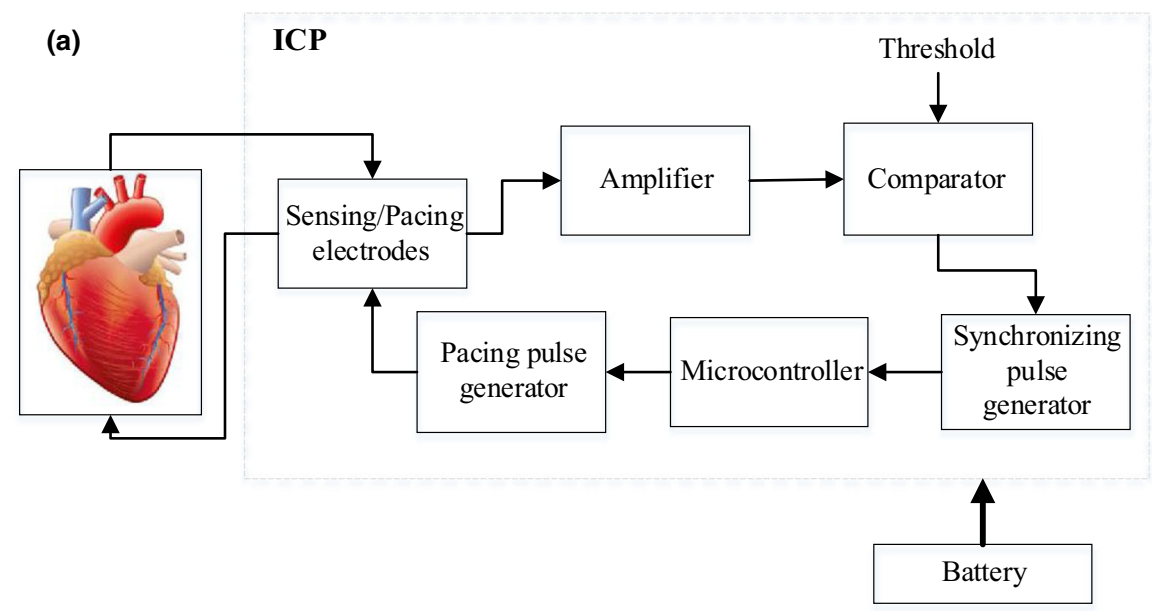

(b)

ICD

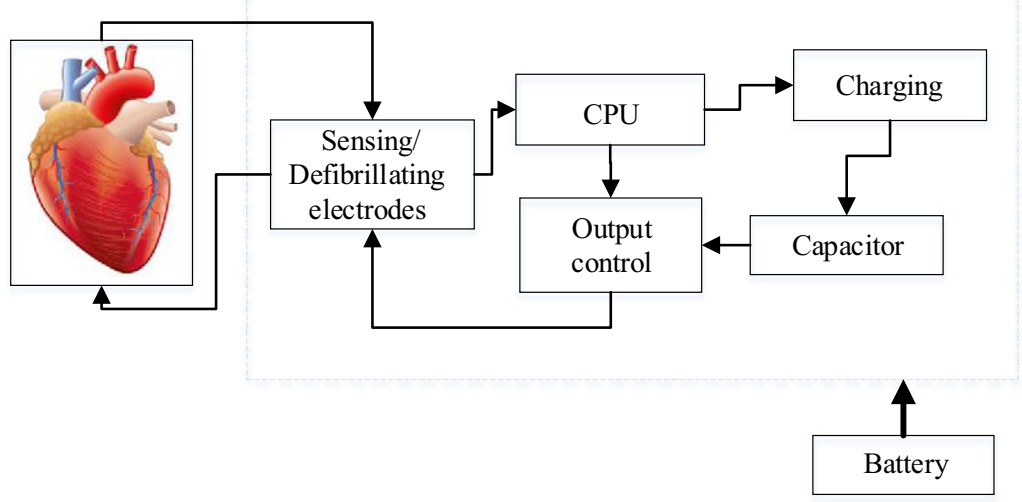

FIG. 1. Schematic representation of an implantable cardiac pacemaker (a) and implantable cardioverter-defibrillator (b) showing the typical subsystems.

TABLE I. Manufacturers of implanted cardiac pacemakers and cardiac defibrillators.

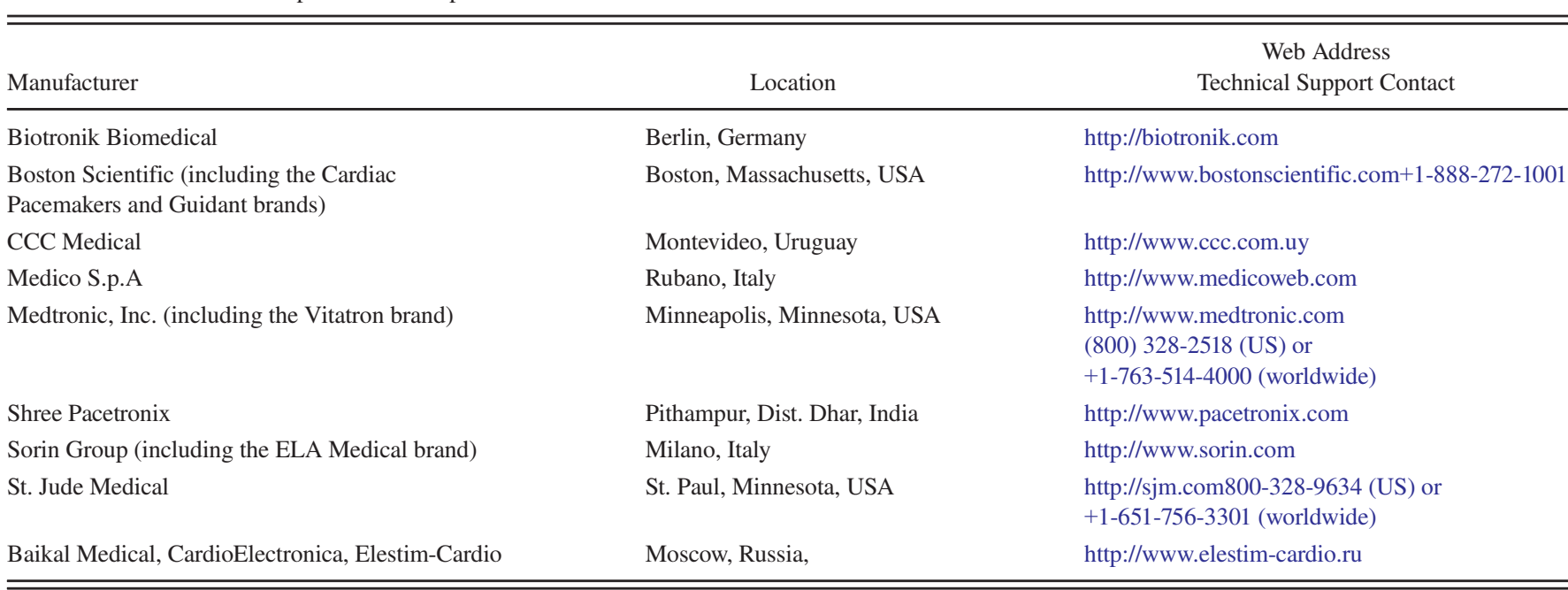

single-chamber device sensing and controlling either the right atrium or right ventricle, or a dual-chamber device sensing and controlling both of these chambers. An ICD includes functions equally as sophisticated as those found in an ICP and adds the capability to sense the onset of a tachycardia or ventricular fibrillation (VF). The ICD also delivers a higher 
voltage pulse to halt such episodes. A typical pacing output is a short duration pulse of 1-5 V and yields output energy of a few tenths of micro-joules. In contrast, the ICD output ranges from a high-speed series of slightly higher voltage pulses to a more than a 700-V shock, delivering between 2 and $35 \mathrm{~J}$ through its auxiliary defibrillation electrodes.

Cardiac implantable electronic devices add an output and lead to pace the left ventricle in order to restore a more physiological timing between right and left heart depolarization. Almost all CIEDs implanted today will include a nonrechargeable power source, an ultra-low-power microcomputer, storage memory, RF communication, specialty power supply, and interface circuitry as shown in Fig. 1. Many devices also include sensors for activity, respiration, temperature, and other factors that can be used to adjust patient therapy. These functions are subject to different interactions with ionizing radiation leading to potentially different and often confusing clinical outcomes.

Complicating matters from a management standpoint, patients may be dependent on their CIED for pacing, or not. However, for CIEDs, pacing dependency is not always clearly defined. A commonly used clinical definition of when pacing is dependent is when patients have an inadequate or absent intrinsic heart rhythm, which turns symptomatic in case of a (sudden) failure of the CIED's pacing function. Symptoms can vary, but may include acute syncope, heart failure, arrhythmia, and can lead to death. ${ }^{35-37}$ The incidence of pacing dependency is roughly $10 \%$. Patients with an ICD can also be pacing dependent. The cardiologist in consultation with the radiation oncologist can categorize the pacing dependency of the patient into one of three classes. Class 1 : patients who display bradycardia-related symptoms that may result in an emergent situation or who have a history of these symptoms in the absence of device pacing (highly dependent). Class 2: patients who are asymptomatic even with an intrinsic ventricular rate of less than 30 beats per minute (intermediately dependent). Class 3: patients with an intrinsic ventricular rate in excess of 30 beats per minute and who have never experienced an emergent situation related to bradycardia (nondependent). In clinical practice, it is difficult to distinguish between the three classes. ${ }^{35-38}$ In RT practice, it is the cardiologist's realm to state the patient's device dependence for the appropriate management during $\mathrm{RT}^{22}$

\section{B. Sensitivities and potential failures}

A number of theoretical mechanisms causing malfunction in these devices from ionizing irradiation have been proposed. One of the principal failure pathways results from an aberrant accumulation of charge or current flow within the irradiated semiconductor material that can induce malfunction. The basic radiation problem is illustrated in Fig. 2 where a CMOS device with normal operation is shown [Fig. 2(a)] in contrast with one experiencing effects from irradiation [Fig. 2(b)]. During normal operation, the gate is isolated from the channel between the source and the drain by a thin layer of silicon dioxide $(\mathrm{SiO} 2)$. The application of the appropriate gate voltage induces the formation of a conduction channel between the source and the drain so that current flows when the device is turned ON. Ionizing radiation produces excess electron-hole pairs in both the silicon and the $\mathrm{SiO} 2$ insulator. When the radiation ceases, the excess pairs in the silicon continue to rapidly recombine, whereas in the oxide layer the electrons flow toward the metal or semiconductor determined by the bias polarity. Then, the holes that escape initial recombination in the valence band are relatively immobile and remain near their point of generation. This buildup results in the trapping of trapped positive charges in the gate oxide [Fig. 2(b)], inducing a negative shift in the threshold voltage needed to be applied to turn the device ON. With a large enough voltage shift the device cannot be turned OFF, even with a zero applied voltage, where the device then fails. The buildup of charge can cause several aberrant behaviors. ${ }^{2} \mathrm{~A}$ detail description of the mechanism of the induced aberrant

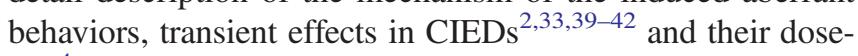
rate $^{4}$ dependence can be found in literature.

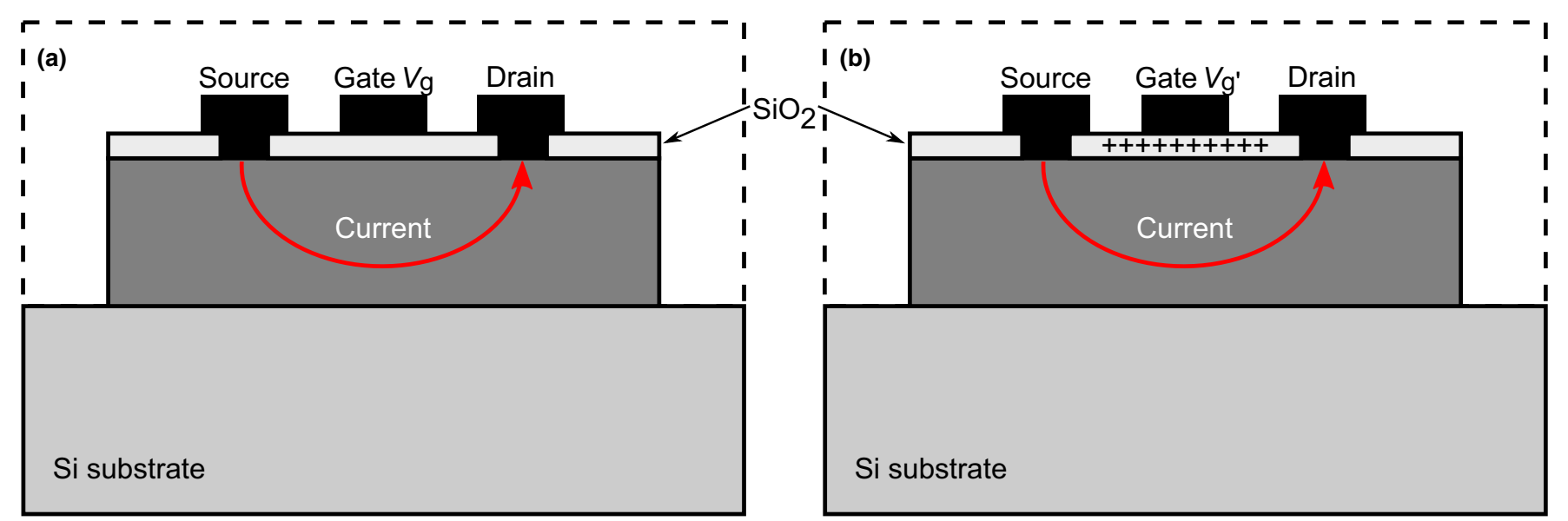

FIG. 2. Schematic diagram of complementary metal oxide semiconductor device illustrating radiation-induced charge, which modifies the threshold voltage. (a) shows the normal operation with current flow for a gate voltage, $\mathrm{V}_{\mathrm{g}}$. (b) shows the buildup of the radiation-induced charge in the $\mathrm{SiO} 2$ layer causing a shift in the threshold voltage, $\mathrm{V}_{\mathrm{g}^{\prime}}$ for current flow. 
Another mechanism for malfunction is the SEU, due to high linear energy (phonton energies $>10 \mathrm{MV}$ ) transfer (LET) ionizing radiation. ${ }^{10,12,29,43}$ Specifically, the small amount of charge stored in the random access memory (RAM) of the device is sensitive to high LET radiation damage, because the electronic state of that RAM (i.e., 0 or 1 ) can be changed by local charge deposition (a high LET is required to deposit sufficient local charge). These consequences are based on a single-event occurrence and are therefore stochastic in nature, with the probability of these events increasing with increasing flux of high LET particles. ${ }^{11}$ This effect is not relevant for the low LET megavoltage electron and photon energies used in RT with LETs less than $0.5 \mathrm{keV} /$ $\mu \mathrm{m}$. However, neutrons produced during high-energy photon (energies $>10 \mathrm{MV}$ ) or proton treatments have sufficiently high LET (1 to $80 \mathrm{keV} / \mu \mathrm{m})^{44}$ to induce this effect.

The device failures described above can manifest clinically in many different ways. These can be broadly described as: not pacing as required by the heart, pacing erratically or inappropriately, complete loss of function, or inappropriate delivery of shocks. These can be further subdivided, as was done by Mouton et al., ${ }^{4}$ as listed below:

1. Missing pulse at the start of irradiation, which is a reversible failure that should have little patient impact,

2. Compatible amplitude modification in which the signal amplitude changes by less than $10 \%$, considered generally as a transient effect that is due to the engineered safety margin in pulse amplitude and should have little clinical consequence,

3. Compatible decreased rate resulting in arrhythmia with average frequency decrease without interruptions longer than $10 \mathrm{~s}$, which may induce lipothymia (light headed/fainting),

4. Compatible accelerated rate resulting in a reversible arrhythmia with a moderate increase in the average frequency,

5. Signal deformation producing permanent defects such as shape distortions of impulses, which may induce incompatible signal changes preventing cardiac stimulation,

6. Amplitude change greater than $10 \%$ is considered to be potentially lethal for patients with a low margin of voltage threshold for cardiac stimulation,

7. Silences longer than $10 \mathrm{~s}$ resulting in an incompatible arrhythmia, which is considered to be potentially lethal,

8. Permanent silence in which there is no detectable signal and no recovery of the signal.

A short summary of the most relevant malfunction modes of CIEDs during RT can also be found in the 2017 HRS consensus report. ${ }^{22}$

\section{CONDITIONS IN CLINICAL USE THAT CAN INDUCE MALFUNCTIONS}

The malfunctions described above in Section 3.B. arise from one of five clinical conditions.

\section{A. Cumulative dose effects}

A high cumulative dose can lead to permanent damage to the device. This is historically the most commonly cited interaction between a CIED and therapeutic radiation. ${ }^{1,2,6}$ The CIED circuitry is degraded in proportion to the accumulated dose often resulting in decreased output amplitude, increased current drain, and erroneous or failed sensor operation, including the heartbeat sensing functions. While some of this degradation may be obvious externally or through the device's telemetry data, there are cases where, for example, with increased current drain, that may not be obvious and can lead to sudden failure immediately or within a few months following therapy. ${ }^{45}$ Such risks make proper assessment and management particularly important, including consideration given to any previous patient radiotherapy. Threshold dose levels that produce damage depend on the manufacturer and model of the device. ${ }^{7}$ It has been shown by various studies that for some new devices, there is no safe radiation dose to avoid all important failures, and some CIEDs have shown failures at 2 Gy or less. ${ }^{4,5,46}$

Mouton et al., ${ }^{4}$ directly irradiated a sample of 96 pacemakers in vitro and showed most failures at cumulative doses of 2 Gy or higher. While the risk of such damage is not stochastic (low doses are not reported to cause damage), individual devices do show a wide variability in their sensitivity to dose failure, making establishment of fixed cumulative dose thresholds challenging. However, it is apparent from the four studies that evaluated direct irradiation of CIEDs that 3 out of 49 devices $(6 \%)$ malfunctioned at a dose $\leq 5 \mathrm{~Gy}$, and 12 out of 38 devices (32\%) malfunctioned at a dose $\leq 50 \mathrm{~Gy} .{ }^{6,47-49}$ Clearly, there is some risk associated with moderate-to-high doses to CIEDs that requires attentive management.

Cumulative dose to CIEDs is most often associated with scattered photons and electrons. For cases of high-energy photon or proton therapy, the cumulative dose added from neutrons can be considered. It is unclear if a radiation weighting factor is appropriate for CIEDs, and if so, what the value would be. However, if biologically based radiation weighting factors are used, the dose equivalent will still generally be much lower than $2 \mathrm{~Sv}$ for both photon and proton therapy. ${ }^{50,51}$ Therefore, cumulative dose estimates can rely on assessment of the photon dose only for high-energy photon therapy. For proton therapy, cumulative dose issues are not generally a concerning unless the device is within the primary proton field.

High cumulative doses have been associated with the following clinical effects in ICPs: (a) changes in pacing pulse (amplitude or pulse deviation changes $>25 \%$ and complete loss of signal which may prevent heart stimulation), (b) sensing threshold changes $>25 \%$, (c) loss of telemetry capability (temporary or permanent) preventing inspection or adjustment of the device settings, (d) pacing frequency changes $>10 \%$ before or after irradiation or device inhibition, (e) complete loss of signal, and (f) miscellaneous effects such as battery depletion and lead impedance changes., ${ }^{92,46,48,52,53}$ Fewer investigational reports are available for ICDs 
comparatively. ICDs are suspected to be more sensitive to radiation than ICPs, due to their more complex circuitry. ${ }^{54} \mathrm{In}$ addition to the failures observed in the ICPs, the existing studies on different ICDs report on sensing threshold changes (as first malfunction), shock, energy deviation $>25 \%$, VT, and even VF that might cause a shock. ${ }^{49}$

\section{B. Neutron-induced upsets}

Neutron-induced upsets in memory or logic circuits can occur. High-LET radiation can change stored values in memory through the SEU mechanism. This may cause changes in the microprocessor circuitry (e.g., resets) without any physical damage to the device ("soft errors"), but may also cause an abnormal high-current state in a device causing loss of functionality ("latch up"). ${ }^{10,11,18,55,56}$ However, even for soft errors where there is no damage to the CIED, it is possible that the upset may not be functionally recoverable in vivo, requiring the device to be explanted ( 2 out of 15 upset events were unrecoverable in the data from Grant et al. ${ }^{57}$ ). Most often, soft errors manifest as a reset of the device or the loss of stored diagnostic data and can be recovered. In some devices, the reset may cause a reversion to default parameters requiring intervention using a pacemaker programmer to restore proper function. In very rare cases, the reset may be delayed for hours or even weeks following the therapy. It is essential that the device manufacturer be consulted for recommendations specific to the implanted device model if neutron presence is anticipated.

Single-event upsets are only associated with high-LET radiation, predominantly neutrons produced during RT delivery. The threshold for this type of interaction in CMOS devices was measured to be about $0.6 \mathrm{keV} / \mu \mathrm{m}$. This is above the LET of therapeutic photons and electrons. ${ }^{58}$ However, it is well below the LET of fast neutrons, which have an LET peak around $10 \mathrm{keV} / \mu \mathrm{m}$ and a maximum value of greater than $100 \mathrm{keV} / \mu \mathrm{m}$. The relationship between LET and a latchup event is complicated. Soft errors are most commonly associated with thermal neutrons, while latch-up events are most commonly associated with fast neutrons or protons (10s of $\mathrm{MeV}$ ).

The risk of a SEU error is stochastic in nature, characterized by a constant probability of failure per unit flux of highLET particles. Consequently, any high-LET radiation exposure to the device is associated with some risk of device failure. As such, it is equally probable that the event will occur at the beginning of treatment as at any later time during therapy (some devices have been described by the manufacturer as contraindicated for RT due to this risk). Over the course of neutron-producing therapy (15 and $18 \mathrm{MV}$ photon or proton therapy), the risk of device malfunction has been found to be between $12 \%$ and $29 \%$ per course of treatment. ${ }^{29,43,57}$ It is preferred to avoid use of protons, and limit photon use to energies less than (or equal to) $10 \mathrm{MV}$ in order to reduce such risks. ${ }^{10,29}$

It is important to note that a device can undergo substantial neutron interactions, even if it is not in close proximity to the treatment site, since secondary neutrons scatter throughout the treatment vault. The risk can only be well mitigated by employing treatments that avoid neutron contamination produced in therapies at energies at or below $10 \mathrm{MV}$. If this is not possible, the risk of reset errors can be reduced by reducing the beam energy, which in turn reduces neutron contamination. Higher treatment energies have higher neutron fluences and therefore will have higher rates of reset errors. The flux at $18 \mathrm{MV}$ is approximately 300 million times greater than the background neutron flux from cosmic radiation $\left(\sim 13 \mathrm{n}^{0} / \mathrm{cm}^{2} \mathrm{hr}\right)$. This flux drops by a factor of 2 at $15 \mathrm{MV}$, and by a further factor of 10 at $10 \mathrm{MV}$. Siemens and Elekta machines both produce less than half the neutrons as compared to Varian accelerators. ${ }^{59-62}$ Beyond considerations of the nominal energy used, most treatment parameters (field size, distance from the field edge, and SSD) have only minor influences on the fluence. ${ }^{61,63}$

While there is some neutron production from 10-MV beams (and even at lower energies), the neutron flux increases dramatically above $10 \mathrm{MV}$. Therefore, in this report, and consistent with the recommendations from other reports, ${ }^{22,57} 10-\mathrm{MV}$ photon beams are considered to be not neutron producing in the context of risk management for patients with CIEDs.

Electron therapy produces few neutrons, even at high energy. A 20-MeV electron beam produces fewer neutrons than a $10 \mathrm{MV}$ x-ray beam (per MU) ${ }^{64,65}$ so electron beams can be considered non-neutron producing. In contrast, proton and heavy ion therapy generate an abundance of neutrons. ${ }^{50,51,66}$ For proton therapy, scanning beam procedures will produce less neutrons than passively scattered therapies, and low-energy treatments will produce less neutrons than high-energy treatments, but all proton and ion therapies should be considered neutron producing. ${ }^{67}$

Attempts to perform neutron dosimetry are not recommended, because stochastic effects are very challenging. Rather, RT procedures should be conducted with low photon energy $(\leq 10 \mathrm{MV})$ or electron beams to avoid neutron contamination. If neutron contamination is unavoidable, the neutron flux should be minimized if possible by using the lowest reasonable treatment energy. Patients should then be managed as described later in this report (Section 8.E).

\section{C. Dose-rate effects}

Dose-rate effects can induce transient interference during irradiation. In some circuits, particularly those associated with cardiac rhythm sensing, reference voltages and physiologic sensors are subject to interference from high dose-rate (Gy/min) x-rays incident on the device's circuits. Additionally, the device can become confused with periodic x-rays signals and interpret them as cardiac signals. This interference, which has also been described during CT imaging, is frequently mistaken for EMI effects. No permanent damage is caused, unless the accumulated dose is also high, but the device may sense inappropriately leading to inappropriate 
ICD shocks, improper or nonexistent pacing output, resets, or other effects. $^{13}$

The severity of failure from dose-rate effects is generally mild, being primarily transient and manifesting as noise while the radiation beam is on. The risk of this failure mode is also relatively low. In a study involving 249 courses of RT, there were only 3 recorded noise events, corresponding to a $1.2 \%$ risk per course of radiotherapy. ${ }^{57}$ While the frequency and severity of this failure mode is much less than the previous two, care should nevertheless be exercised to minimize the risk of patient harm when the device is exposed to a high dose-rate, even if it does not result in a high total dose. ${ }^{4}$

Although the vast majority of dose-rate effects tend to be both transient and manageable, this may not always be the case. Important failures due to dose-rate effects can include: (a) amplitude changes of electrical signal $>10 \%$, (b) silence in electrical signal $>10 \mathrm{~s}$, and (c) permanent silence (no signal). These failures may be potentially lethal for a pacemakerdependent patient. ${ }^{46}$ Also of concern is that the risk of these effects in clinical devices is not well documented. Mouton et al., ${ }^{4}$ is the only study to date to examine dose-rate dependency. While they concluded that the most important failure probability ( $70 \%$ of irradiated pacemakers) is observed for $8 \mathrm{~Gy} / \mathrm{min}$, whereas no pacemakers failed at a dose-rate lower than or equal to $0.2 \mathrm{~Gy} / \mathrm{min}$, these devices already had a high cumulative dose $(6.2 \mathrm{~Gy})$ before the dose-rate was increased to $8 \mathrm{~Gy} / \mathrm{min}$. Consequently, dose-rate effects cannot be isolated from cumulative dose effects in these data. This study also found one irradiated pacemaker that exhibited an important defect at a dose-rate of $0.2 \mathrm{~Gy} / \mathrm{min}$, with a cumulative dose of only 0.15 Gy. However, this study used 18-MV photons, so it is highly probable that the observed effect at the low dose-rate was actually due to SEU from neutrons. Clearly, dose-rate effects have not been well separated from cumulative dose and SEU effects in the literature, and have also often been erroneously described as EMI effects. Therefore, dose-rate issues are highly uncertain. Matters are further complicated, because the relationship between risk of failure and dose-rate is not simple or even monotonic. There can be complex interplay between the dose-rate, the frequency of dose pulses, and the sensing band of a CIED. It is not known which time-scale is most relevant for dose-rate effects: dose per linear accelerator (LINAC) pulse (elevated in flattening filter free "FFF" beams), dose per second (elevated for higher nominal dose-rates), dose per minute (elevated in volumetric modulated radiation therapy "VMAT"), or dose per fraction (elevated in stereotactic body radiation therapy "SBRT" or stereotactic radiosurgery "SRS"). ${ }^{68}$

In an effort to provide some guidance on this issue, there have been several recommendations to maintain a dose-rate of $0.2 \mathrm{~Gy} / \mathrm{min}$ or less. ${ }^{4,5,7,10,46}$ Some manufacturers recommend dose-rates $<0.01 \mathrm{~Gy} / \mathrm{min}$ however, as this dose-rate has not been found to increase the risk of device error. A $0.01 \mathrm{~Gy} / \mathrm{min}$ dose-rate (or higher) could be exceeded in clinical practice if the device is within $5 \mathrm{~cm}$ from the field edge (for conventional therapy). ${ }^{69}$ For intensity-modulated radiation therapy (IMRT), slightly more caution may be warranted.
IMRT delivery yields higher leakage radiation doses and dose-rates. Although IMRT treatments are also typically slower, the risk depends on whether the relevant dose-rate is of the level dose/s or dose/min, which is not well known with dynamic delivery. However, there is no published evidence currently available that suggests that the slightly different dose-rates (e.g., from IMRT/VMAT, SBRT, or even specifically using FFF beams for therapy) result in an elevated risk to CIEDs. Given that the bulk of experience is based on conventional dose-rates, increased monitoring (pre- and posttreatment) may be necessary when hypofractionation is used, in agreement with the 2017 HRS expert consensus. ${ }^{22}$ Especially, if the patient would benefit from a different dose-rate offered by IMRT/VMAT, SBRT, or FFF beams, such an approach is almost certainly appropriate given the low risk from dose-rate effects and the limited data ${ }^{70}$ indicating an elevated risk.

Dose-rate considerations are a potentially greater concern for imaging procedures. For short-duration imaging procedures, high dose-rates may still be acceptable provided they do not occur for a long period of time ( $>3 \mathrm{~s}$ ). Such long periods can be insufficient time for transient effects to manifest clinically. The $\mathrm{kV}$ planar images only last a short time and therefore are unlikely to confuse the CIED. MV portal images or simulation CT scans will not only exceed the $0.01 \mathrm{~Gy} / \mathrm{min}$ dose-rate but they are also typically delivered very quickly (less than few seconds), and are therefore well tolerated. However, CBCTs deliver $>0.01 \mathrm{~Gy} / \mathrm{min}$ dose-rate over a relatively long period of time. If the device is within the field of view (or even within 1-2 cm of the field of view), it may receive high dose-rates for a long time with a risk of misssensing. Similar concerns exist for extended-duration CT scans, such as 4DCT procedures. A mechanism that has been recently documented ${ }^{71}$ occurs with CT scanners. CT scanners typically rotate at approximately twice per second, resulting in maximum dose to the device with a period of approximately 120 pulses per minute. This signal can be misinterpreted as a physiological heart rate and trigger a device response. In general, LINACs deliver pulses in a mode much more rapidly than that of $\mathrm{CT}$, making it less prone to be confused for a cardiac signal.

In summary, if the dose-rate to the device is below $0.01 \mathrm{~Gy} / \mathrm{min}$ (e.g., it is outside $5 \mathrm{~cm}$ of the edge of the treatment field, or $2 \mathrm{~cm}$ in the longitudinal direction of a CBCT or 4DCT FOV), published research to date concludes there is little risk to the patient. However, if the dose-rate is above this, further management is warranted as described in Section 8.E.

\section{D. Magnetic fields effects}

The effects of MR imaging procedures on CIEDs vary and are thoroughly described in the HRS 2017 report. $^{22}$ They depend on several factors including the device type, how the device is programmed, the static magnetic field strength, and the imaging conditions. Strong MRI magnetic fields may cause the following effects: 
1. Lead tip heating - radiofrequency currents can be induced in the pacing lead resulting in damage to tissue near the electrode,

2. Unintended stimulation - radiofrequency currents from MRI can cause pulsed energy to be emitted from the lead tip, potentially causing stimulation to tissue and unintended pacing,

3. Mechanical movement - the static and time-varying magnetic fields can induce mechanical forces causing the device to move (force and torque),

4. Radiofrequency interference - radiofrequency energy during the MRI procedure can be coupled into the circuitry producing transient interference with sensitive circuits such as the sensing amplifier,

5. Undesired artifacts - imaging of normal tissues and target volumes may exhibit degraded resolution.

\section{E. Electromagnetic interference (EMI)}

Induced effects from EMI may result in sensing the field as myocardial potential resulting in the inhibition of the output. ${ }^{2}$ Other potential malfunctions may include: (a) shut-off of the reed switch (electrical switch operated by applied magnetic fields sensor susceptible to EMI) that would result in fixed pacing rate, (b) triggering of output, or (c) isolated serious permanent disruption of function and inappropriate reprogramming. ${ }^{2}$ EMI effects have not been well documented in the literature, and in particular have not been well isolated from other malfunction mechanisms. Even so, the sensitivity of CIED devices to EMI has been reported to be minimal and of transient nature, existing only while the interference is present. ${ }^{46,52}$ Electromagnetic noise around modern LINACs is minimal. There is little concern with transient effects, when present, for CIEDs. ${ }^{6}$ Possible reprogramming of an ICD has been reported, as a poorly understood interaction with the LINAC or the radiation beam itself. ${ }^{9,10,52,53}$ In today's RT practice, other sources of EMI for CIEDs remain uninvestigated for some electromagnetic image guidance systems, respiratory gating technologies, and more recently developed treatment modalities.

\section{MALFUNCTION RISK ASSOCIATED WITH CLINICAL PROCEDURES}

The following sections discuss the potential source of malfunction for the various imaging and therapeutic procedures used in RT.

\section{A. Imaging}

\section{A.1. $k V$ Imaging for treatment planning and localization}

Several recent studies have shown a small risk to CIEDs from CT irradiation. ${ }^{13,14}$ The most common effect observed was oversensing. ${ }^{13,14}$ Over-sensing in ICPs may result in an inappropriate inhibition of pacing output, with the additional complication of possibly triggering unnecessary tachyarrhythmia therapy in ICDs. ${ }^{22}$ Based on these studies, the effects were transient and were only observed during direct irradiation of the CIED generator. Effects were not observed when the leads were irradiated. ${ }^{13,14,72}$ Transient effects that last only a short period of time, such as over the course of a traditional CT scan, do not generally last long enough to induce clinically relevant effects. However, Hayes et al., ${ }^{73}$ suggested that clinically significant inhibition could result from pauses in excess of $3 \mathrm{~s}$ for patients that are dependent on their CIED. As such, brief CT examinations are safe for patients with a CIED. Caution may be warranted when performing prolonged scans such as perfusion imaging or 4DCT to manage risk to the device. However, CT irradiation of a CIED has not shown to be a clinically significant problem. ${ }^{74}$ For positron emission tomography with 2-deoxy-2-[fluorine18] fluoro-D-glucose integrated with CT (18F-FDG PET/CT) scans, patients with a CIED can safely undergo the PET scan. However, patients should inform the technologist about the presence of the CIED at the time of the scan. Imaging a CIED may result in an uptake of FDG update around it, potentially increasing the risk of false positive or false negative readings by the radiologist.

In addition to oversensing, the cumulative dose delivered to the device can be considered. For a multislice adult chest CT, typical CT doses range from $0.03-0.05 \mathrm{~Gy},{ }^{75,76}$ while the dose from a 4DCT scan is approximately eight times greater. ${ }^{77}$ Still, even these doses are small for individual scans. Image guidance procedures also tend to yield low doses. If intensive imaging is used regularly, cumulative doses (particularly in combination with the dose from the therapeutic procedure) may become relatively high. Elekta X-Ray Volume Imaging (XVI; Elekta, Crawley, UK) and Varian On-Board Imaging (OBI; Varian, Palo Alto, CA, USA) systems yield a dose of typically $0.001-0.003$ Gy per radiographic image. These procedures are short in duration, so there is little risk from dose-rate effects. However, fluoroscopic dose-rates of $0.0108 \mathrm{~Gy} / \mathrm{min}$ are possible with the Elekta $\mathrm{kV}-\mathrm{CBCT}{ }^{78}$ Fluoroscopic imaging times range from $30 \mathrm{~s}$ to $5 \mathrm{~min}$, with imaging doses exceeding $0.1 \mathrm{~Gy}$ for a 5 min fluoroscopic study. ${ }^{79}$ Similarly, for the Varian CBCT system, cumulative doses can easily be $0.09 \mathrm{~Gy}$ and typically take $1 \mathrm{~min}$ to deliver. ${ }^{80}$ A compilation of local and effective doses from $\mathrm{kV}-\mathrm{CBCT}$ for the surface and center of the head and chest are provided in Task Group 75. ${ }^{81,82}$ While the cumulative dose will often be small, the doserates may be high and therefore of concern when coupled with the relatively long duration of many of these procedures. Assessing the risk to radiation is challenging, because of the unclear relationship between dose-rate and CIED effects. However, the reported "over-sensing" effect, where the CT scan signal is mistaken for a cardiac signal, would clearly not be possible for fluoroscopic or CBCT procedures. It is for this reason that fluoroscopic or CBCT project to be relatively safe procedures comparatively. The scientific community would clearly benefit from improved understanding of this issue. 


\section{A.2. MV imaging for treatment localization}

There are three major modes of MV imaging: portal imaging, MV-CBCT (Siemens, Erlangen, Germany), and Tomotherapy helical MVCT (Accuray, Sunnyvale, CA, USA).

Each imaging session using port films produces a dose between 0.01 and $0.05 \mathrm{~Gy} .{ }^{81}$ Typical exposures during a single MV-CBCT image acquisition range from 0.02 to 0.15 Gy (although lower doses are becoming more achievable ${ }^{83}$ ), and with the dose close to being uniformly distributed. ${ }^{84}$ One advantage of using MV-CBCT beams is that they can be modeled in a treatment planning system and the dose can therefore be accounted for in the treatment plan. ${ }^{85-87}$ The tomotherapy imaging system produces helical MVCTs resulting in maximum imaging doses of $0.01-0.02 \mathrm{~Gy} .{ }^{88}$

Dose-rates associated with MV imaging should be considered similar to those from $\mathrm{kV}$ imaging. Port films are short, with transient effects almost certainly not clinically relevant. Longer MV CT scans may yield a high dose-rate for an extended period of time. The risks associated with such conditions are not well studied. However, the lack of data indicating a problem suggests such procedures are relatively safe. Cumulative doses from MV imaging procedures should be considered in the management of patients with CIED devices as they may accumulate substantially over the course of treatment. Of particular note is that imaging fields tend to be somewhat larger than the treatment fields. This can result in the delivery of the full imaging dose to the CIED device. Treatment planners should be encouraged to consult with the radiation oncologist to confirm if smaller setup fields could be used to minimize the dose to the CIED.

\section{A.3. MR imaging}

Performing MRI scanning for patients with a CIED has been an issue of extensive debate. ${ }^{89-91}$ Historic guidelines for safe MRI scanning strongly discourage MR examination of CIED patients, except in case of urgent need, as documented by the American Heart Association. ${ }^{92}$ However, in the past few years, a number of studies have shown that MRI can be done safely on patients with an ICP or ICD, ${ }^{90,91,93}$ culminating in a report largely focusing on this topic by the Heart Rhythm Society. ${ }^{22}$ This report highlighted that with approximately 1500 MRI scans involving MR-conditional devices (i.e., those for which an MR environment does not pose a known hazard) only one serious adverse event occurred. Even over the course of hundreds of MRI scans of CIEDs that were not MR conditional, the risk of CIED malfunction was not significantly elevated (low) compared to CIEDs that were not exposed to magnetic fields. There were nevertheless many reported instances of CIED malfunctions associated with magnetic fields (particularly resets and transient effects). This report recommends that a protocol for performing MRI safely on patients with CIEDs be implemented based on a well-established multidisciplinary procedure. Particularly for nonconditional devices, while MRI scanning may not be avoidable, it should be done with caution as there may be abandoned leads still present.

\section{B. Therapy}

\section{B.1. $k V$ therapy treatment units}

During $\mathrm{kV}$ therapy, devices near the treatment fields will receive dose from collimator scatter, head leakage, and internal scatter. Dose levels outside the treatment field from these procedures are less well documented than for MV treatments, which may necessitate in vivo dose estimates. Otherwise, concerns associated with $\mathrm{kV}$ therapy are similar to those from MV therapy described below.

\section{B.2. Brachytherapy}

Similar to kV therapy, dose levels distant to brachytherapy sources are not well documented and may require in vivo dose estimates. While the dose to the device has been measured to be low from clinical procedures including coronary artery brachytherapy, ${ }^{94}$ the dose may also exceed $1 \mathrm{~Gy} .{ }^{95}$ For example, the Mammosite ${ }^{\circledR}$ (Hologic, Inc., Marlborough, MA, USA) system can deliver 1 Gy at $15 \mathrm{~cm}$ from the source (for a prescription dose of $34 \mathrm{~Gy}$ ), although Croshaw et al., reported a maximum dose of 1.03 Gy from a balloon breast applicator with an average distance to device of $9.1 \mathrm{~cm}$ from three patients (with no adverse event). ${ }^{96}$ Optimization flexibility may allow for dose reduction during multilumen treatment for partial breast irradiation. ${ }^{97}$

A recent study ${ }^{98}$ made use of a simple method of estimating the maximal dose to the implanted CIED from an HDR spherical breast applicator surface. Based on Table II, one can evaluate the expected maximal dose to a CIED prior to treatment. For a CIED at close proximity to the breast applicator, a more sophisticated plan optimization and dose evaluation is likely appropriate. It was shown that the iodine contrast medium in the balloon reduced the dose to the device up to $9 \% .^{99}$ In the absence of a CIED and other inhomogeneities, the agreement between this study and the previous one ${ }^{98}$ was within $2 \%$.

The accuracy of dose calculation to the CIED is complicated by the high effective atomic number of the device leading to increased dose deposition as compared to water. For

TABLE II. Estimated maximal dose $\left(D_{d}\right)$ to the device for typical distances from a spherical breast applicator's surface. (Table reproduced from Kim et al. $\left.^{98}\right)$.

\begin{tabular}{lcccccc}
\hline \hline Balloon Vol. (cc) & 35 & 50 & 70 & 90 & 110 & 125 \\
\hline Balloon Diam. (cm) & 4.06 & 4.58 & 5.12 & 5.56 & 5.94 & 6.20 \\
1 Gy & 15.6 & 16.9 & 18.2 & 19.3 & 20.2 & 20.8 \\
2 Gy & 10.5 & 11.3 & 12.1 & 12.8 & 13.4 & 13.8 \\
3 Gy & 8.2 & 8.8 & 9.4 & 10.0 & 10.4 & 10.7 \\
4 Gy & 6.8 & 7.3 & 7.8 & 8.2 & 8.6 & 8.9 \\
5 Gy & 5.9 & 6.3 & 6.7 & 7.1 & 7.4 & 7.6 \\
\hline \hline
\end{tabular}

Estimated dose to the device at distance, $\mathrm{d}$ from a spherical applicator is given by: $D_{d}=D_{P} \cdot\left(\frac{R_{\text {ballon }}+1}{R_{\text {balloon }}+d}\right)^{2}$, where $\mathrm{D}_{\mathrm{P}}$ is $3.4 \mathrm{~Gy}$ at $1 \mathrm{~cm}$ from applicator surface and $\mathrm{R}_{\text {balloon }}$ is the balloon radius (cm). 
example, for balloon-to-device distances of 5-10 cm, Monte Carlo calculations have found that the dose is elevated by a factor of approximately 2.4 as compared to typical measurements or clinical calculations. ${ }^{99}$ However, as detailed in Section 4.B, given the substantial uncertainties associated with this correction, there is ambiguity concerning the beam quality on which manufacturer-recommended dose tolerances have been based. That research recommended using standard measurements or calculation techniques to determine dose, with additional corrections for the effective-Z of the CIED not recommended. Similarly, intraoperative RT is another modality available that has been reviewed in a case report where a low cumulative dose did not induce any effects. ${ }^{100}$

\section{B.3. MV photon and electron Beams}

Cardiac implantable electronic devices devices should not be in the direct radiation field of therapy beams if possible, because of the possibility of loss of function by the device. This may be particularly important for ICDs which have shown interference as soon as they were placed inside the radiation field. ${ }^{77}$

Various studies on direct and indirect irradiation of CIEDs have been performed with a wide range of results both on the type and severity of malfunctions, and on the minimum absorbed dose to the device that caused that malfunction. Tables III and IV summarize recent in vitro and in vivo ICP and ICD studies, respectively. ${ }^{46,52}$ Additionally, the Heart Rhythm Society has published a consensus statement on the management of CIEDs based on all types of malfunctions caused by all known sources with complete set of recommendations and extensive literature of all the reported cases of interaction of radiation with cardiac implantable devices. ${ }^{22}$ Special consideration is warranted for relatively new treatment modalities.

Photon therapy treatment (out-of-field doses): The radiation dose outside of the treatment field decreases approximately exponentially with increasing distance from the treatment field edge. ${ }^{101}$ At $10 \mathrm{~cm}$ from the field edge, the radiation dose is approximately $1 \%$ of the target dose. Dose outside the treatment field increases with field size, but is generally independent of energy. Patient scatter contributions to the out-of-field dose are reduced for IMRT in contrast to conventional therapy, because of a reduced treatment volume. However, head leakage contributions are elevated in proportion to the modulation of the beams. Overall, the impact of IMRT on the total dose to the CIED is highly dependent on the specific treatment plan parameters and relative location of the device to the treatment field edge. ${ }^{102-105}$ A separate consideration for stray radiation outside the treatment field is that the out-of-field dose near the surface is elevated relative to dose below the surface, by as much as a factor of 5. ${ }^{105,106}$ This elevated dose is maximal at the surface, and decreases until a depth of dmax, beyond which the dose is relatively constant. ${ }^{69,103,105,106}$ CIED devices are typically located from 1 to $3 \mathrm{~cm}$ below the skin.

In addition to the photon leakage and scatter contributions, high-energy treatments (>10 MV) also generate neutrons. These neutrons are produced in the head of the accelerator, ${ }^{50,104,107}$ and subject the patient to a broad and

TABLE III. CIEDs in vitro studies in recent literature.

\begin{tabular}{|c|c|c|c|}
\hline Souliman $^{42}$ & 1994 & $\begin{array}{l}18 \text { ICPs (dual- and single- } \\
\text { chamber models, various } \\
\text { manufacturers) }\end{array}$ & 11 ICPs failed with no recovery, first compete failure at accumulated dose of $16.8 \mathrm{~Gy}$ \\
\hline Mouton $^{4}$ & 2002 & $\begin{array}{l}96 \text { ICPs (various } \\
\text { manufacturers and models) }\end{array}$ & $\begin{array}{l}66 \% \text { amplitude change }>10 \%, 50 \% \text { permanent silence, } 48 \% \text { slowed down rate, } 41 \% \text { silence longer than } \\
10 \text { s. } 30 \% \text { accelerated rate, } 27 \% \text { missing impulses at start of irradiation, } 23 \% \text { signal compatible } \\
\text { modification, } 17 \% \text { shape deformation.NB. } 5 \% \text { of all pacemakers were concerned with multiple short } \\
\text { silences, which are considered to be harmful. }\end{array}$ \\
\hline Hurkmans $^{6}$ & 2005 & 11 ICDs (4 manufacturers) & $\begin{array}{l}\text { First malfunction observed at } 0.5 \mathrm{~Gy} \text {. Sensing threshold changes were often seen as first malfunction. } 4 \\
\text { ICDs failed } \leq 2.5 \mathrm{~Gy}, 4 \text { ICDs showed VT or VF, which would have resulted in delivery of a shock. No } \\
\text { significant changes in pulse amplitude or pulse frequency were observed. No telemetry problems } \\
\text { recorded. }\end{array}$ \\
\hline Hashimoto $^{128}$ & 2012 & 4 ICDs (same manufacturer) & Proton beam with secondary neutrons-29 Soft Error (SEU) were noted, 1event/15 Gy approx. \\
\hline Hashii ${ }^{108}$ & 2013 & $\begin{array}{l}8 \text { ICDs ( } 2 \text { models same } \\
\text { manufacturer) }\end{array}$ & 10- and 18-MV photon beams, no hard errors observed, soft error more frequently observed at $18 \mathrm{MV}$. \\
\hline Zaremba ${ }^{34}$ & 2013 & $\begin{array}{l}10 \text { ICPs and } 2 \text { ICDs } \\
\text { (various manufacturers) }\end{array}$ & $\begin{array}{l}6 \text { and } 18 \mathrm{MV} \text { to } 150 \mathrm{~Gy} \text { ( } 2 \text { Gy fraction) dose.6-MV group: One ICP malfunction; } 18-\mathrm{MV} \text { group: } 14 \text { ICP } \\
\text { malfunctions at lower dose. No ICD malfunctions were noted at any dose level. }\end{array}$ \\
\hline
\end{tabular}


TABLE IV. CIED in vivo studies in recent literature

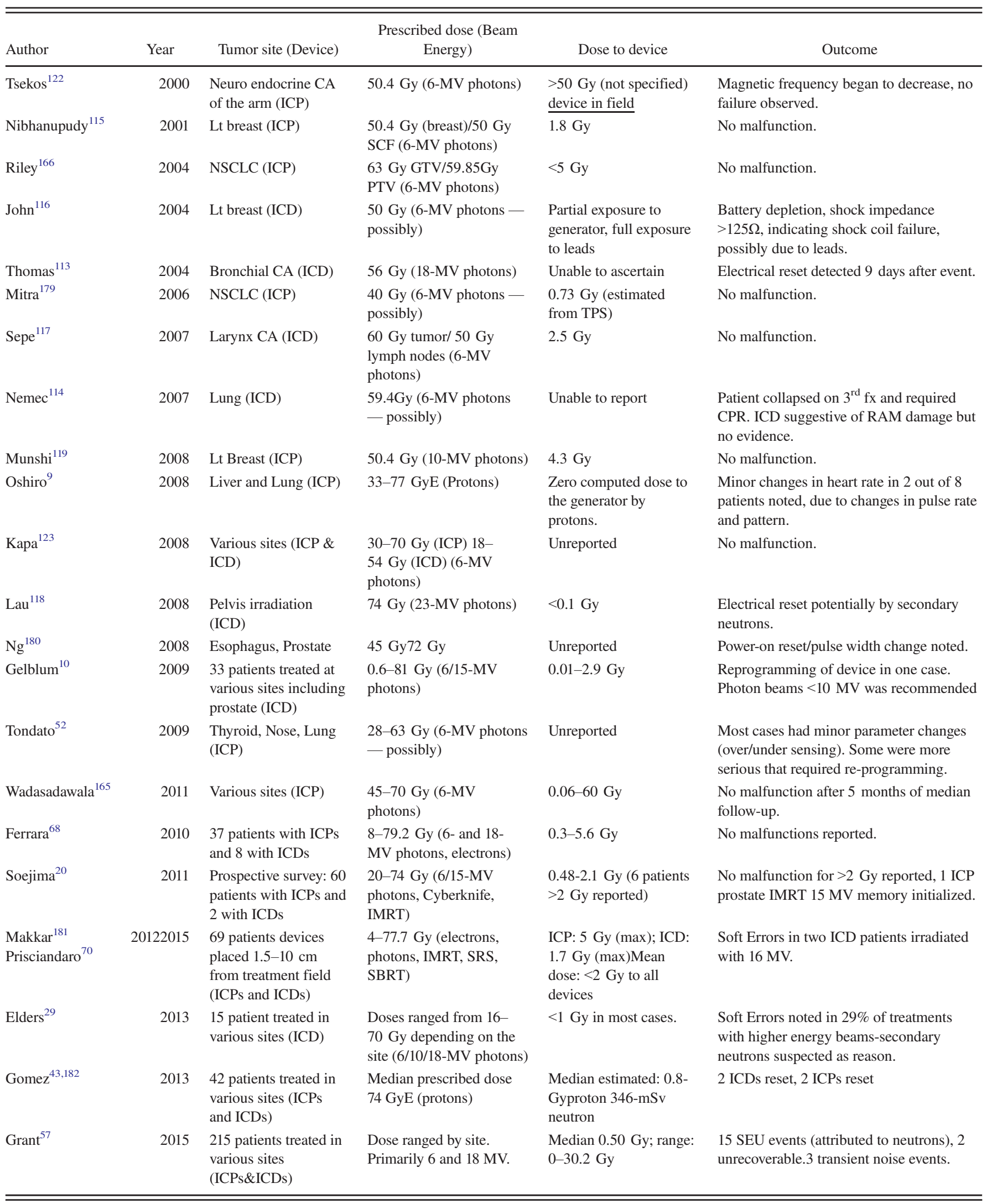

GTV: gross tumor volume; PTV: planning target volume; SCF: supraclavicular field; NSCLC; nonsmall cell lung CA; TPS: treatment planning system; GyE: gray equivalents; VF: ventricular fibrillation; VT: ventricular tachycardia. 
relatively uniform bath of neutrons that may affect the device. ${ }^{108}$ Neutron fluence and absorbed dose (inside and outside the treatment field) increases with nominal $\mathrm{x}$-ray energy, and varies with accelerator manufacturer (being highest for Varian LINACs) ${ }^{59,62}$ Neutron absorbed dose changes minimally with field size ${ }^{62,107}$ or distance from the field edge, ${ }^{105}$ but does decrease rapidly with depth. ${ }^{50,109}$ It is important to note that if shielding is desired, high- $\mathrm{Z}$ materials provide little protection against neutrons. Instead, hydrogenous materials are required for attenuation. ${ }^{110}$ Since electronic devices can show substantial sensitivity to high-LET radiation, ${ }^{111}$ any neutron contribution should be carefully considered.

IMRT: The vast majority of studies in the literature have not specified the treatment delivery technique $e^{10,68,112-114}$ or have merely used $2 \mathrm{D}$ or $3 \mathrm{D}$ techniques. ${ }^{17,115-119}$ One institu$\operatorname{tion}^{120}$ looked at 24 patients with CIEDs treated for localized prostate cancer using IMRT. All devices were interrogated prior to treatment delivery and one week after treatment completion. It was determined that six ICPs suffered from an interference. Each device reset to the manufacturer's default settings. In addition, two ICPs permanently remained in the default settings. Although no mention of beam energy was made in the study, ${ }^{120} 18 \mathrm{MV}$ beam is suspected. One additional reference used 6-MV IMRT for thyroid carcinoma metastatic to the right cardiac atrium and ventricle of an ICPdependent patient with the leads in the treatment field. For this research, no effects to the devices were noted. ${ }^{121}$ In another study that included IMRT, two partial reset events were identified, but concluded to be related to the high photon energy (16 MV) used. ${ }^{70}$ In order to obtain a better understanding of this sensitivity, authors who publish case reports of CIED malfunctions in the future should provide more details on the irradiation technique and conditions than have been reported in the past. Furthermore, an in vitro study of sensitivity of CIEDs to scatter dose from any sort of dynamically modulated delivery is of future interest.

Stereotactic Body RT (SBRT): Stereotactic body RT is a unique modality, because of the potential for long treatments and high dose-rates (e.g., FFF beams). However, the cumulative dose to the device will typically be smaller than for filtered beams. Given the high dose per fraction, length of the treatment session, number of treatment beams, high dose-rates, and beam-on-time for SBRT, caution should be exercised when treating patients with CIEDs. The treatment planner should avoid using beam directions that pass through the device. Planning and delivery parameters should be carefully inspected in terms of the number and energy of the beams, beam directions, dose-rate, MU per fraction, MU/control-point for VMAT, beam-on-time, and treatment time, as this may affect the dose received by the CIED. Due to these special considerations, such patients should be managed as discussed below in Section 8 .

Although direct irradiation of the CIEDs should be avoided in clinical SBRT practice, it is important to emphasize that malfunctions can also occur by scattered radiation outside the treatment field. ${ }^{118,122,123}$

Electron therapy treatment (out-of-field doses): Electron RT also results in dose outside the treatment field. ${ }^{124,125}$ Although, the dose is often much lower than that from x-ray therapy. Elekta and Siemens accelerators in particular can have higher scattered doses than from x-ray therapy than for Varian LINACs. ${ }^{65,125}$ Out-of-field radiation is electrons, and therefore not very penetrative, so the dose is predominantly limited to the patient surface on which the electron beam is incident (i.e., for an anterior electron field, the anterior patient surface receives the bulk of the out-of-field dose). However, CIEDs are superficially implanted, and the out-offield electron dose has been measured to be as high as 3\% of the given dose at dmax, extending at this level to beyond $30 \mathrm{~cm}$ from the field edge. ${ }^{126}$ The cumulative doses for superficial locations in the patient could potentially reach as much as 2 Gy. The cumulative out-of-field dose from electron therapy should therefore be assessed if the CIED is on the same local surface of the patient as aimed at by the treatment field, and up to $30 \mathrm{~cm}$ from the treatment field edge.

Neutron production with electron beams is less than that from a $10 \mathrm{MV}$ x-ray beam. ${ }^{64,65}$ While high-energy electron beams may be problematic for the most sensitive of electronic systems, electron beams can broadly be considered to be "non-neutron producing".

\section{B.4. Neutron and proton/particle beams}

Neutron therapy treatment: High LET particles produce densely ionizing tracks that are more effective in causing damage than low LET radiation, and a high cumulative dose is not required. ${ }^{12}$ Koivunoro et al., ${ }^{53}$ showed that epithermal neutrons in boron neutron capture therapy (BNCT) produced severe malfunctions in ICPs at doses between 1 and 2 Gy. It is often recommended not to treat CIED patients with neutron therapy. Even outside the treatment field, there will be an abundance of high-LET neutrons that could adversely affect the CIED.

Proton and heavy ion therapy treatment: These modalities also produce an abundance of secondary neutrons. However, high-energy photons and passively scattered protons have 1-2 orders of magnitude higher neutron production (depending on distance from field border) than scanned protons and scanned carbon beams ${ }^{127}$ (see Fig. 3). An experimental study on the influence of proton and charged particle beams on CIEDs placed outside the irradiation field showed that the frequency of power-on reset (SER) errors observed was twice as high for ICDs in the proton beam irradiation than in carbon-ion beam irradiation. ${ }^{128}$

For passive scattering systems, neutrons are generated in the treatment head, beam modulators, scattering devices, and patient-specific apertures or compensators, and are the 


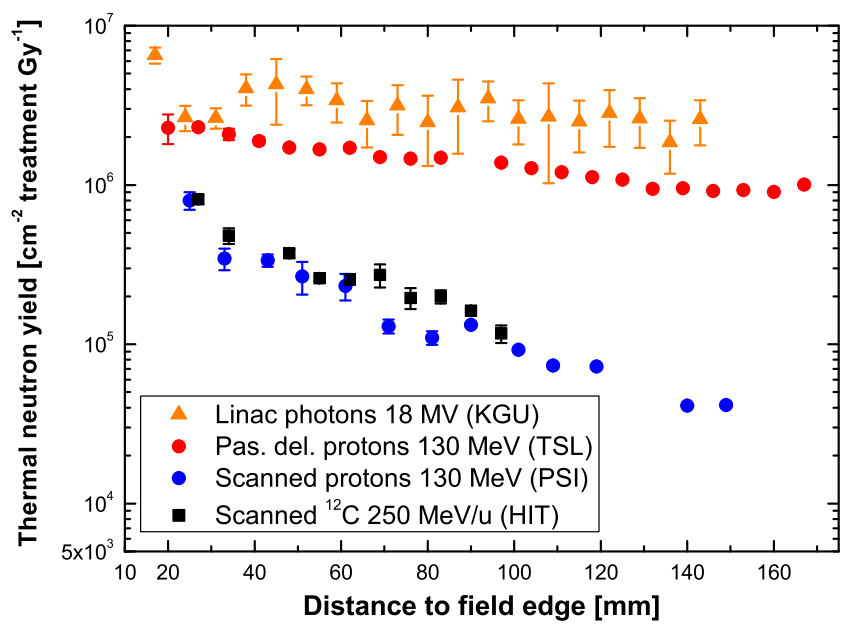

FIG. 3. Fluence of secondary thermal neutrons for all radiation types and delivery techniques in the depth of maximum dose $(31 \mathrm{~mm}$ for $18 \mathrm{MV}$ photons and $125 \mathrm{~mm}$ for charged particles) during the irradiation of the water phantom with a $5 \mathrm{~cm} \times 5 \mathrm{~cm}$ field. (Reprinted from Kaderka et al. with permission). ${ }^{127}$

dominant contribution to the total dose in the region downstream of the Bragg peak and at distances greater than 10-15 $\mathrm{cm}$ from the field edge. ${ }^{129}$ The field defining aperture dominates as a secondary neutron production source due to its proximity to the patient, making the neutron dose dependent on the ratio of the field size to the aperture opening. ${ }^{66}$ Out-of-field patient neutron doses increase with beam range/energy and treatment volume. ${ }^{66,130}$ Active/modulated scanning systems do not require scattering devices in the treatment head or patient apertures. Consequently, the secondary neutron production in the treatment head is reduced leaving the majority of neutrons generated by the patient's body. ${ }^{6,129,131}$ Although scanning systems have a smaller out-of-field equivalent dose compared to passive scattering systems by a factor of 40 in the superficial region, this factor decreases at deeper depths. ${ }^{129}$

Based on device interrogation, minor ICP malfunctions such as changes in pulse rate and pulse patterns were noticed in 2 out of 8 patients (all patients were device dependent more than $80 \%$ of the time). ${ }^{9}$ Therefore, electrocardiographic monitoring was recommended for all patients with an ICP during proton beam therapy. ${ }^{9}$ A more recent phantom study on four ICDs, outside the treatment field of a passive scattering proton irradiation, ${ }^{128}$ found:

1. The gamma ray dose was approximately 0.000110.00045 Gy per Gy of protons, with a ratio of gamma ray dose at central axis of $5 \%$ of the total secondary dose (unimportant effect on the ICDs),

2. The major contribution of secondary out-of-field dose was due to neutrons with 0.0027 per Gy of protons for the ICDs and 0.0013-0.0089 Gy per proton Gy, for the phantom,

3. There was $1 \mathrm{SEU}$ per $15 \mathrm{~Gy}$ and 1 power-on reset error per 50 Gy and,

4. The timing in occurrence for soft and hard errors was unpredictable and random.

\section{DOSE ASSESSMENT CONSIDERATIONS}

The dose (and dose-rate) delivered to the CIED is of particular importance in managing patients with implanted devices. The dose to the device can be crudely estimated from literature such as $\mathrm{TG}-36^{69}$ or $\mathrm{TG}-158{ }^{132}$ If the device receives a dose that is concerning (i.e., $>2$ Gy), further evaluation is warranted prior to treatment. In general, if the device is further than $10 \mathrm{~cm}$ from the photon treatment field edge, the dose will not exceed 2 Gy. However, if it is within $10 \mathrm{~cm}$, a more rigorous determination of the dose is appropriate (e.g., a measurement or calculation, see Section 6.C). The precision of any dose assessment should be sufficient to ensure that the patient is properly categorized and therefore properly managed.

There are several dose assessment considerations that merit attention.

\section{A. Where to assess dose}

While some areas of the device are less sensitive than others, these may not be obvious and will vary between different devices. Furthermore, it is not possible to determine how the device is seated in various patients. Therefore, the most prudent and reasonable approach is to assess (at least) the maximum dose to any point in the device. Of note, CIED leads ("heart wires") will not be damaged by clinically relevant doses ${ }^{72,121}$ (for electronically passive leads where mechanical damage would be the greatest risk). Such leads do not need to be considered as part of the CIED, since they are highly resistant to therapy doses. However, ongoing research is investigating electronically active leads (which are metal wires emanating from a CIED that contain additional electronic components at the distal end for monitoring the performance of the device) that could be damaged by radiation. It is not known whether they should be considered as part of the CIED. The most practical option is therefore to include them until this issue is better understood.

\section{B. Medium specification}

Careful understanding is required when using the phrase, "dose to the CIED". Dose measurements (and many calculations) are typically reported as dose-to-water or muscle and as such, dose reporting is almost always specified to one of these media. Yet, neither are present in electronic devices. The predominant mechanism for device damage is the buildup of trapped charge in the device's insulating layers leading to shifts in transistor operating parameters. Correspondingly, a dose to $\mathrm{SiO}_{2}$ is more relevant than dose to tissue. (Unfortunately, even this is insufficient and even more complicated. While a dose to silicon predicts the rate of free charge generation, the final amount of trapped charge is also dependent on the radiation energy and circuit operating conditions.) Consistent with the higher effective atomic number of $\mathrm{SiO}_{2}$, interaction cross sections are higher than in tissue, 
particularly for low-energy photons $(<0.1 \mathrm{MeV})$. Thus, the traditional "dose" (as in dose-to-water) will be an underestimation of the dose to the device, particularly outside the treatment field where the spectrum is softer. ${ }^{133}$ Although not directly quantified, $\mathrm{SiO}_{2}$ would be expected (based on similar know use of $\mathrm{Al}_{2} \mathrm{O}_{3}$ ) to overrespond relative to tissue by $\sim 20$ $30 \%$ outside of a 6-MV treatment field, ${ }^{134}$ and potentially by more than a factor of 3 for $\mathrm{kV}$ energies. ${ }^{135,136}$ Ultimately, accurately calculated tissue dose serves as a good estimate of dose to the device inside the megavoltage treatment field, but is an increasingly poor estimate out of the beam or in $\mathrm{kV}$ environments. This task group does not recommend accounting for it. We recommend to report measured doses per their normal clinical calibration, where no additional corrections need to be applied, even to the treatment planning system (TPS) calculations. This recommendation was based on: (a) the overresponse of $\mathrm{SiO}_{2}$ in clinical situations not being well established, (b) the relationship between dose to $\mathrm{SiO}_{2}$ and consequences of its use not being straight forward, (c) dose tolerances promulgated by the manufacturers are often based on beams of unknown energy, but may include low-energy beams (in which there would be no overresponse relative to the conditions in which the dose limit was established), (d) a potential $20-30 \%$ dose error is believed to be within the acceptable uncertainty of dose determination to the device outside the RT field and, (e) all published data on the radiation effects on these devices are reported in dose-to-water or tissue already. However, for imaging intensive IGRT procedures this estimate more seriously underestimates the dose (potentially by a factor of 3 or more). The terminology of "dose to the CIED", as used in this report and in history, should be understood to typically refer to "dose-to-water" or to something similar at the location of the device, rather than truly as dose to the device.

\section{C. Dose assessment}

\section{C.1. Calculation}

A convenient option for assessing the dose to the CIED if it is near (or in) the treatment field is to use the treatment planning system. Particularly close to the treatment field, the TPS is likely the most accurate way to assess dose. This is because steep dose gradients make measurements very challenging. Treatment planning systems can readily estimate the maximum dose to the device. Caution must be used still, because treatment planning systems routinely underestimate the dose outside of the treatment field. ${ }^{137-139}$ Even for a simple conventional field, ${ }^{137}$ the treatment planning system typically underestimates the actual delivered dose. This calculation error can be $40-125 \%$ at $4-11 \mathrm{~cm}$ from the field edge. The treatment planning system is useful if the CIED is within $3 \mathrm{~cm}$ of the treatment field edge (or within the 5\% isodose line for an IMRT plan) where reasonable dose calculations are achievable. Beyond $3 \mathrm{~cm}$ from the treatment field, the TPS should not be used as a method of assessing dose to the device.
Calculation options exist for CIED devices located more than $3 \mathrm{~cm}$ from the edge of the treatment field. For example, AAPM TG- $36^{69}$ or Peridose ${ }^{140}$ are accurate within approximately $30 \%$ (underestimating) ) $^{140-142}$ on average. However, these systems are limited to conventional fields and do not incorporate IMRT treatments. Monte Carlo models have also been developed that are capable of calculating the dose outside of the treatment field with good accuracy for arbitrary treatments and geometries. ${ }^{103,105,143}$ Nevertheless, development of such models is demanding and requires extensive coding and validation. ${ }^{103,144}$

Neutron contamination is not included in any commercial treatment planning system, nor is it handled by TG- $36^{69}$ or Peridose. ${ }^{140}$ For neutrons, the cumulative dose is generally unimportant. Only the presence or absence of neutrons has been reported to matter.

\section{C.2. Measurement}

Generally, beyond $3 \mathrm{~cm}$ from the treatment field a measurement is the most appropriate way of determining the dose to the CIED. The maximum dose to the CIED should be measured, corresponding to the point of the device that is closest to the treatment field and most superficial. Dosimeters with high sensitivity are recommended. Dose measurements are less accurate outside the treatment field, because of greater uncertainties in the characteristics of the radiation field. Two major considerations are justified when considering radiation field characteristics: energy and depth of treatment.

Energy: For measurements outside the treatment field, a substantial portion of the dose comes from low-energy scatter radiation. The energy spectrum of a 6-MV photon beam averages only $200-400 \mathrm{keV}$ outside the treatment field, vs. $1.5 \mathrm{MeV}$ inside. $^{133,145}$ This will result in an overresponse of many dosimeters in terms of determining dose to tissue for out-of-field measurements. ${ }^{146}$ Although the relevant comparison may better be described as dose to $\mathrm{SiO}_{2}$, as discussed in Section 6.B., it is recommended to report dose-to-water when possible. Therefore, dosimeters with a relatively flat energy response or well-known corrections are ideal. The energy response is particularly important for patients treated with orthovoltage energies or treatments involving IGRT, as the overresponse of common dosimeters can exceed a factor of 3 at $\mathrm{kV}$ energies. ${ }^{135,136}$ Barring a flat energy response or welldocumented correction factors, dosimeters can be calibrated against an ion chamber in an out-of-field geometry. Ion chambers show little energy dependence in this energy range with simple correction. ${ }^{133}$ Calibration should be done at $1 \mathrm{~cm}$ depth and at $5 \mathrm{~cm}$ or more from the field border. Dosimeter results should then be related to their readings under standard reference open field $(10 \mathrm{~cm} \times 10 \mathrm{~cm})$ calibration conditions.

Depth: The depth of the CIED requires attention for patient-specific measurements. Outside of the primary field, the dose is highly elevated (by a factor of 2-5) at the surface and there is a build-down effect until a depth of $d_{\max }$ is reached. ${ }^{103,106}$ Therefore, bolus should be used over the 
detectors to provide buildup to match the depth of the CIED. Devices are typically implanted at a minimum depth of $0.5 \mathrm{~cm}$, but are often placed deeper in the muscle under layers of body fat. If the device is superficially implanted, or the depth is unknown, dosimeters on the patient's surface should be placed at a depth of $0.5 \mathrm{~cm}$. If the device is implanted at a known depth, the measurement should be made at that depth (up to the beam's nominal $d_{\max }$ ). Although a depth of $1 \mathrm{~cm}$ is a practical solution, it mitigates the bulk of this build-down effect.

Several detectors suitable for CIED dosimetry are discussed below.

Ion Chambers can be used in phantom to assess the dose, and typically have only small corrections. A major exception is the use of microchambers with high- $Z$ electrodes that can show dramatic overresponse to low-energy photon beams. ${ }^{147}$ Caution must also be used for in vivo measurements, because of the necessary high voltage.

Thermoluminescent dosimeters (TLD) are well suited for the measurement of dose to CIEDs. TLD use is detailed in the upcoming TG-191 report, ${ }^{148}$ including special considerations for out-of-field applications. Although most out-of-field considerations are minor, the soft out-of-field photon spectrum causes an overresponse of less than $10 \%$. Correction factors are available when soft beams are considered. ${ }^{133} \mathrm{Cau}-$ tion must be used with the standard LiF TLD-100 at high xray energies $(>10 \mathrm{MV})$. This dosimeter overresponds to thermal neutrons. While in-field measurements are reliable, measurements outside the treatment field can be in error by more than a factor of $10 .{ }^{141}$ For out-of-field dosimetry in high-energy photon beams, the TLD-700 is recommended for use. It has a very low thermal neutron cross section, ${ }^{149}$ but will accurately measure only photon doses.

Optically Stimulated Luminescent Dosimeters (OSLDs) are expected to be detailed in an upcoming AAPM TG-191 report. OSLDs are reasonably well suited to measurement of the dose to CIEDs as they can be read-out $10 \mathrm{~min}$ following irradiation and with little loss of signal. As they have a relatively high effective atomic number, OSLDs can overrespond to the soft spectrum outside of the treatment field by more than $25 \%$. Correction factors are also available for these detector types. ${ }^{134}$ Similarly, OSLDs overrespond in the $\mathrm{kV}$ range by a factor of 3 or more. ${ }^{135,136}$ Their overresponse to $\mathrm{kV}$ radiation can be accounted for by a calibration at the approximate $\mathrm{kV}$ energy. While OSLDs have shown some angular dependence, this effect is on the order of a few percent for most situations relevant to clinical therapeutic RT, ${ }^{150}$ and is therefore considered irrelevant for CIED dosimetry.

Diodes have the required sensitivity, but overrespond to lower energy scatter radiation. The magnitude of this overresponse varies between devices, but can be $70 \%$ or more at just $1 \mathrm{~cm}$ outside the treatment field. ${ }^{145}$ Other corrections may also be appropriate, such as dependence on angular orientation, temperature, and dose-rate, etc. These effects are typically small. For example, dose-rate dependence can approach $10 \%{ }^{151}$ The variety and culmination of these correction factors can be a challenge for out-of-field dosimetry, although if the device is well characterized this dosimeter may readily provide sufficiently accurate dose measurements.

Metal oxide semiconductor field-effect transistors (MOSFETs) can provide an immediate dose measurement, and have properties similar to diodes regarding energy, temperature, angular dependence, and dose-rate dependence. However, their sensitivity is limited to about $0.10 \mathrm{~Gy}$. The MOSFET also has a finite lifetime of about $10 \mathrm{~Gy}$ accumulated dose. In addition, these detectors are more expensive than other in vivo dosimeters. Special consideration may also be required in an IGRT environment, as MOSFETs can overrespond by a factor of $\sim 3$ to $\mathrm{kV}$ photons. ${ }^{152}$

Film can also provide a dose measurement to the CIED. Film can be cut to provide a two-dimensional estimate of the CIED dose. However, since the out-of-field radiation spectrum has a number of lower energy photons and since film has a relatively higher atomic number, radiographic film will have a substantial over-response. In contrast, radiochromic film will provide a more accurate estimate of the CIED dose, because its atomic number is closer to water. ${ }^{153}$

Table $\mathrm{V}$ provides a summary of characteristics of commonly used detectors used for megavoltage in vivo dosimetric measurements. While we have ample options for dosimeter usage, dosimeters continue to be developed. For example, scintillators have minimal energy dependence and may be well suited to dosimetry for CIED devices. ${ }^{154,155}$

Neutron dosimetry is particularly challenging and requires specialized equipment and expertise as detailed in the TG158 report. ${ }^{132}$ Therefore, neutron dosimetry is not recommended unless both equipment and expertise exist. When neutron production is unavoidable, it should be minimized by using the lowest suitable treatment energy. Furthermore, the patient should be monitored in the High-Risk category (see Table VI).

\section{C.3. Typical out-of-field doses}

Out-of-field dose estimates are presented in TG- $158^{132}$ for a range of treatment modalities. In the context of a $2 \mathrm{~Gy}$ threshold, the following general observations can be made. For conventional photon therapy, and a 50-60 Gy tumor dose, the dose to the CIED will be less than 2 Gy for most scenarios if the device is more than $5 \mathrm{~cm}$ from the field edge. For IMRT treatments, the dose could exceed $2 \mathrm{~Gy}$ if the device is within $\sim 7 \mathrm{~cm}$ from the field edge. This distance may be slightly smaller for FFF beams ${ }^{156,157}$ or VMAT techniques, ${ }^{158}$ but likely only minimally. This distance may also be slightly smaller for SRS/SBRT modalities ${ }^{157}$ (in part due to lower target doses), although noncoplanar beams may increase the dose to the device. Therefore, as a simple rule of thumb, for external beam x-ray therapy, if the device is within $10 \mathrm{~cm}$ of the field edge (or within $10 \mathrm{~cm}$ of the $50 \%$ isodose line for an IMRT treatment), the dose to the device should be determined (e.g., in vivo measurement if the device is between 3 and $10 \mathrm{~cm}$ from the field edge, or for IMRT, a TPS calculation if the device is within $3 \mathrm{~cm}$ of the field edge or $5 \%$ isodose line should be specifically performed). If the 
TABLE V. Summary of characteristics of commonly used detectors used for megavoltage in vivo dosimetric measurements. ${ }^{183}$ While any detector listed may be able to achieve the necessary precision, the dual requirements of high sensitivity and relative energy independence for out-of-field measurements lend preference to TLDs and OSLDs.

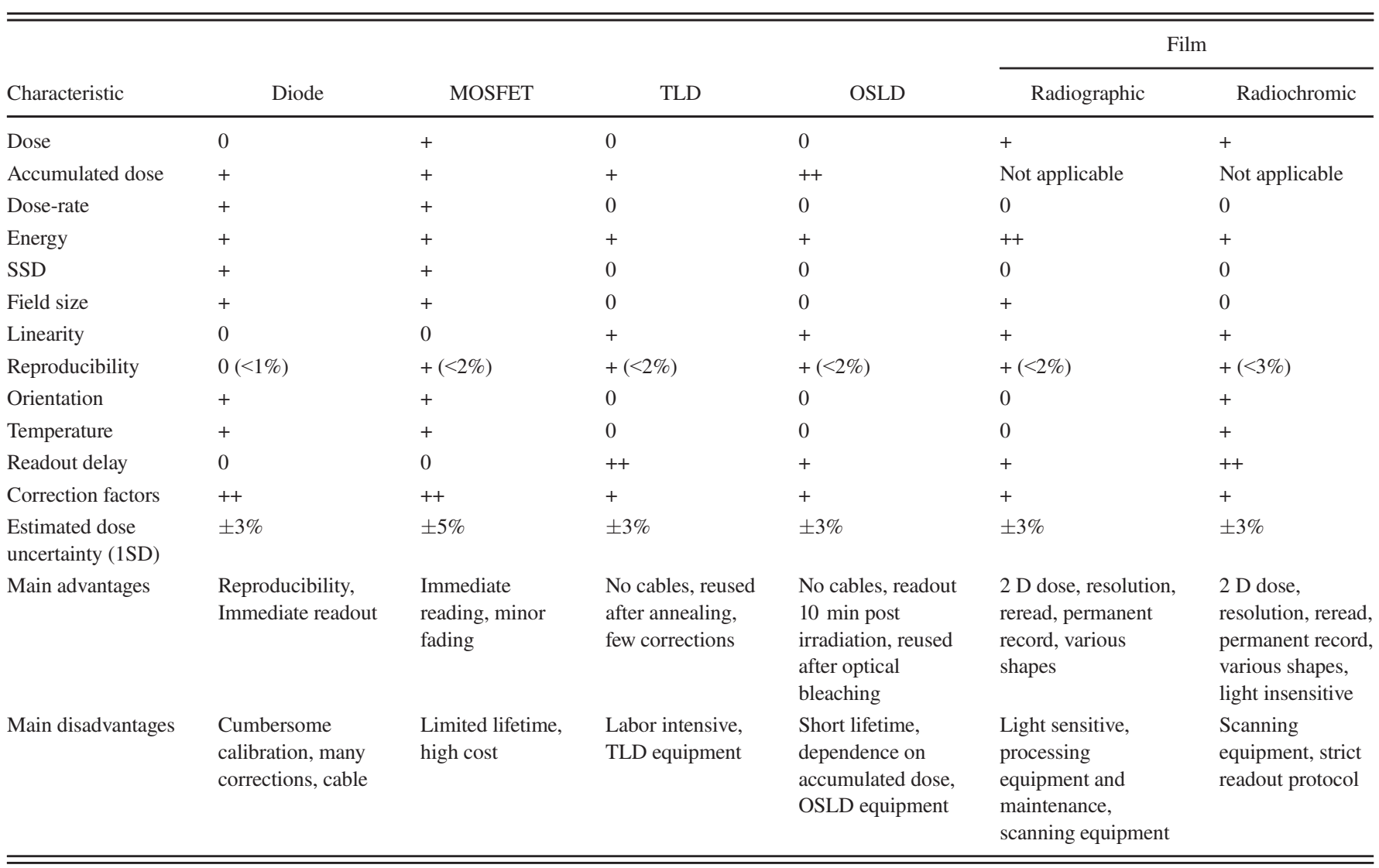

0 , No concern; +, Minor concern; ++ , Serious concern.

Assumes calibrations at a particular energy.

Orentation plays a role in readout.

For measurements within the radiation field where their response has been well characterized. Values based on the authors' experience and publications on in-field measurements (Mijnheer et al. 2013). ${ }^{183}$ As discussed in the section on out-of-field measurements, the uncertainties would be increased. The reader is referred to TG- $158^{132}$ for a discussion on detector uncertainties for out-of-field measurements.

Assumes a well-maintained processor.

TABLE VI. Patient risk categories: cumulative dose to the CIED and pacing independent versus pacing-dependent. The patient's risk is not equal to the risk of a CIED defect. Higher cumulative doses can be used if the manufacturer recommended dose tolerances are above the dose thresholds presented here. Neutrons are considered present when protons or photons with energy $>10 \mathrm{MV}$ are used.

\begin{tabular}{lcccc}
\hline \hline Patient & \multicolumn{4}{c}{ Dose region and risk category } \\
\cline { 2 - 5 } & $<2$ Gy & $2-5$ Gy & $>5$ Gy & $\begin{array}{c}\text { Neutrons } \\
\text { present }\end{array}$ \\
\hline $\begin{array}{l}\text { Pacing } \\
\text { independent } \\
\begin{array}{l}\text { Pacing } \\
\text { dependent }\end{array}\end{array}$ & Low risk & Medium risk & High risk & High risk \\
\hline \hline
\end{tabular}

device is entirely more than $10 \mathrm{~cm}$ from the field edge or $10 \mathrm{~cm}$ outside of the $50 \%$ isodose cloud for IMRT treatments, a dose assessment is not necessary unless noncoplanar/vertex beams are used, as in the case for a Cyberknife treatment. If noncoplanar beams are used, dose verification should be considered even at CIED distances beyond $10 \mathrm{~cm}$ from the treatment field edge.

Treatments in proton therapy will typically have less than 2 Gy of neutron dose. Neutron dose measurements are not recommended, because of the challenges in dosimetry and the variability of data in the literature. Rather, these patients should be monitored in the High-Risk category on the basis of the risk for a single-particle SEU.

\section{RECOMMENDED DOSE THRESHOLDS}

From the preceding sections, it is evident that issues related to the device, beam energy, and dose play an important role in determining the chance of device malfunction that can arise during RT. It is also evident that there is limited information available to define a quantitative dose-effect relationship within a narrow confidence interval. The only dose threshold available otherwise is a zero dose threshold. The absence of any dose given yields no effects on the CIED. Above this threshold, there is an increasing chance of a 
device malfunction. There are no published data showing any detrimental radiation effects in these devices at doses less than $0.5 \mathrm{~Gy}$ in photon beams with energies $10 \mathrm{MV}$ or lower.

The type of device malfunction, being transient, reversible, or irreversible, is not predictable when considering only the cumulative dose received by the device, the dose-rate, or the presence of high-LET radiation. Permanent damage to the device has been repeatedly documented from high cumulative doses or the presence of high-LET radiation. The risk of these different outcomes is important when trying to decide what type of precautions should be taken to minimize a possible device malfunction and what type of action is needed, should such an event occur. Furthermore, patient-related factors should also be taken into account. A patient who is not pacing-dependent might require less monitoring compared to a patient who is pacing dependent. Similarly, the indication for RT is also important. The small risk of a device failure is likely acceptable for a patient referred for RT, because of an acute paraplegia risk. Finally, the available cardiology infrastructure and knowledge will influence how patient management can be established in a specific institution.

To summarize, these are the factors that are considered in the proposed recommendation:

1. CIED type, model, and whether it contains active leads,

2. Presence of neutrons or other high-LET radiation, which might cause SEUs,

3. Cumulative dose, which might lead to permanent failures,

4. Patient's pacing dependency,

5. Dose-rate at the location of the CIED, which might lead to mostly transient oversensing effects or reset type events,

6. Radiation therapy treatment intent (curative/palliative).

Weighting these considerations is not a mathematical exercise. This report's aim was to define an optimally safe and practical procedure that can be implemented on a large scale. As such, it is important to prioritize the recommendations based on clinical relevance. The patient's pacing dependency mainly determines the immediate impact of the CIED malfunction on the patient, whereas the cumulative dose and the presence of high-LET radiation produces the most abundant (and often severe) malfunctions. These aspects are combined to derive patient risk categorizations for levels of Low Risk, Medium Risk, and High Risk (see Table VI).

The relative importance of different dosimetric factors (cumulative dose, presence of high-LET neutrons, and doserate) is very complex and will vary by device type, clinical practice, and specific treatment conditions. A large-scale review of 249 courses of RT in CIED patients sheds some light on this in clinical practice as it included courses that delivered $>2$ Gy to the device, and courses that included neutron-producing radiation (usually $18 \mathrm{MV}$ photons). Grant et al. ${ }^{57}$ observed 18 event malfunctions, including 15 SEUs ( 2 of which were permanent malfunctions) and 3 transient (and asymptomatic) noise events. The risk of SEU based on cumulative dose and neutron-producing RT is shown in Fig. 4, illustrating the risk of failure in clinical practice overwhelmingly due to neutrons. Research shows that neutronproducing therapy is more clinically common than having the device receive a high cumulative dose. In the study by Grant et al, ${ }^{57} 71$ out of 249 patients (29\% of cases) were treated with neutron-producing therapy. In contrast, cumulative doses greater than 2 Gy were seen in 47 cases (19\%), and greater than 5 Gy was seen in only 4 cases (2\%). Of the cases treated with neutron-producing therapy, $21 \%$ had a risk of SEU device malfunction during the course of therapy. None of the devices that received a high cumulative dose suffered any observed effects. These limited data were the justification by the HRS 2017 report $^{22}$ that prevented them from recommending a cumulative dose threshold or monitoring strategy for high cumulative doses. In an ex vivo setting, four studies evaluated direct irradiation of CIEDs and reported that $6 \%$ of

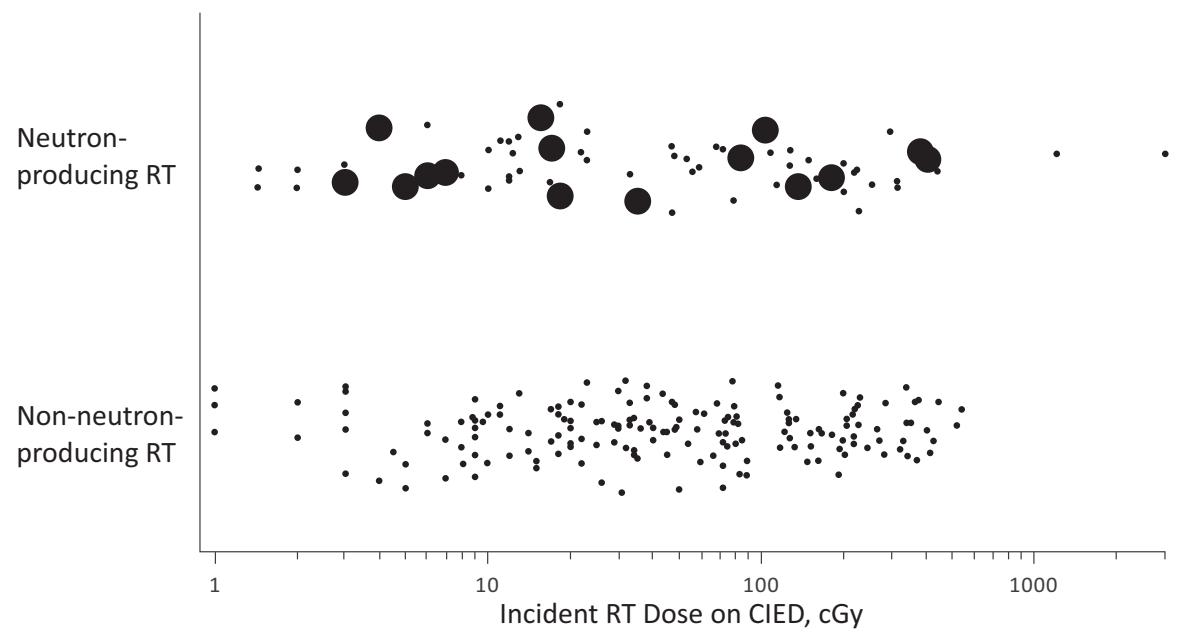

FIG. 4. Incident RT dose received by the CIED, grouped by the neutron-producing capability of the RT. Neutron-producing RT included 15-MV and 18-MV photon beams; non-neutron-producing RT included $6 \mathrm{MV}, 10 \mathrm{MV}$, Co-60, and electron beams. Large dots identify courses in which a SEU took place. Note that dose is plotted on a logarithmic axis. Reproduced from Grant et al. ${ }^{57}$ 
devices malfunctioned at a dose $\leq 5$ Gy with $32 \%$ malfunctioning at a dose $\leq 50 \mathrm{~Gy} .{ }^{6,47-49}$ At high CIED doses, particularly above $5 \mathrm{~Gy}$, the risk of device malfunction is apparent and comparable to the risk associated with neutron-producing therapies. Therefore, it is reasonable that in cases of high cumulative dose, the CIED should be monitored in a comparable manner as in cases of neutron-producing therapy.

In the study by Grant et al, ${ }^{57}$ there were also 3 noise events observed out of the 249 courses of radiotherapy which occurred in 2 out of 215 patients. All of these events were observed with less than 0.5 Gy delivered to the device and attributed to dose-rate effects.

Use of secondary neutron-producing radiation is one of the more important considerations when categorizing risk to the device. Given the very high rate of failures in neutronproducing beams, patients treated with any neutron-producing beams should be considered as High-Risk. As for cumulative dose, there are limited data (see Tables III and IV) that support modification of the 2 Gy dose threshold originally proposed by the AAPM TG- $34^{1}$ to define risk categories. Therefore, we have also adopted this dose level for Low-Risk patients, unless the device vendor recommends a higher dose threshold that should then be followed. While modern technology reprogrammable CIEDs that incorporate advanced CMOS circuitry have reported an increase in radiation sensitivity, ${ }^{7,46,52}$ other investigators argue against the modern CIEDs' increased sensitivity to radiation. ${ }^{54}$ There is currently no definitive evidence that new technology CIEDs are more or less sensitive to radiation than the older bipolar semiconductor technology ones ${ }^{54}$ or that ICDs are more or less sensitive to radiation than ICPs. ${ }^{43}$ Only a minority of the patients are pacing dependent. It then follows that only a minimum level of monitoring should be provided for the majority of patients.

There was not consensus among the task group members to either adapt the treatment, revise the position the CIED, or have ECG-monitoring for all patients who were expected to receive CIED doses of more than 2 Gy. Such measures would only be warranted for patients receiving a higher CIED dose. The HRS 2017 expert consensus report ${ }^{22}$ has a very useful discussion on device repositioning, highlighting the important risk associated with moving the device, ultimately recommending against moving the device for Low-Risk scenarios (i.e., cumulative doses below 5 Gy). Thus, a second upper dose threshold of $5 \mathrm{~Gy}$ is introduced by this report, which would only apply to a small subset of patients, above which more intensive monitoring is warranted. These dual thresholds make the guideline easier to implement and better aligned with clinical practice. The dose threshold of $5 \mathrm{~Gy}^{22}$ is more stringent than the $10 \mathrm{~Gy}$ threshold recommended by the new Dutch and Italian practice guidelines. ${ }^{159,160}$ This threshold was chosen, because the number of patients that will fall in the category of 5 Gy or higher was deemed manageable (see Table VI). Additionally, there are very few reports of CIED malfunctions at doses less than 5 Gy (see Tables III and IV). Some vendors (e.g., Medtronic $^{161}$ ) recommend dose threshold of 5 Gy to their devices already. ${ }^{159}$ In addition, the introduction of our 2-5 Gy dose level does not duplicate the 5 Gy value discussed in the 2017 HRS expert consensus report. ${ }^{22}$ We explicitly recommend determining the dose delivered to the device as well as a management strategy for both device dependent and independent patients (see next section). Another reason for introducing the intermediate dose level is that there is limited data available from only a finite number of vendors that "allow" 5 Gy to the CIED. There are vendors that recommend 3-5 Gy dose thresholds, with others still recommending $<2$ Gy (e.g., Biotronik). If the cumulative dose is estimated to be 5 Gy or more, adaptation of the treatment plan can be considered. Even at these high doses, the risk and consequence of device failure are likely smaller than the risk from suboptimal radiotherapy.

\section{PATIENT MANAGEMENT AND RECOMMENDATIONS}

Key aspects of the patient management workflow are presented in Fig. 5 for consideration and guidance.

\section{A. Staff and department requirements}

Prior to treating patients with CIEDs, the RT department should develop a treatment management plan in cooperation with the cardiology department or cardiologist's office. A treatment management plan may involve numerous people in different capacities, with clear role definitions and communication pathways. A sample workflow, including the suggested roles and responsibilities of each individual, is provided in Fig. 6. At least one radiation oncologist, one qualified MP, and one nurse knowledgeable on the function of CIEDs need to be involved to ensure the correct and safe management of patients about to undergo RT. Assistance from a cardiologist or CIED technician is necessary to provide ad hoc consultation. Radiation therapists should receive specialty training for the management of CIED patients from the qualified MP. The RT department should consider opportunities to have appropriate personnel available for monitoring these patients. The institution should develop specific guidelines for the management of radiotherapy patients with a CIED as well as be able to recognize and assist with CIED-related problems as they arise. The RT department is responsible for training the therapists as well as ensuring the availability of specialty equipment (Table VII). At a minimum, the training should include understanding the purpose of the device, what visual and audio monitoring is required, what typical problems a patient might exhibit during treatment, and who to notify if the patient exhibits any of these problems. When an ICD patient is scheduled to undergo RT, a device expert from cardiology or the device clinic should be consulted as to whether switching the ICD anti-tachycardia therapy OFF is appropriate. This can be accomplished with a heavy magnet or by a properly trained device technician who can program this function $\mathrm{OFF}$ and $\mathrm{ON}$ at each fraction. A standalone RT center without a cardiology department should consider coordinating with the device vendor to have a technical 


\section{Patient with CIED and indicated Radiotherapy}

- Inform treating cardiologist and inform patient

- Determine patients' pacing-dependency

- Inform if anti-tachycardia therapy can be switched off by magnet when ICD.

- Estimate dose on CIED

o More than $10 \mathrm{~cm}$ from treatment field edge - dose probably $<2 \mathrm{~Gy}$

$\circ$ Less than $10 \mathrm{~cm}$ from field edge - measure to confirm dose

- Within $3 \mathrm{~cm}$ from field edge - can rely on TPS calculation

- Minimize dose to CIED with treatment plan optimization

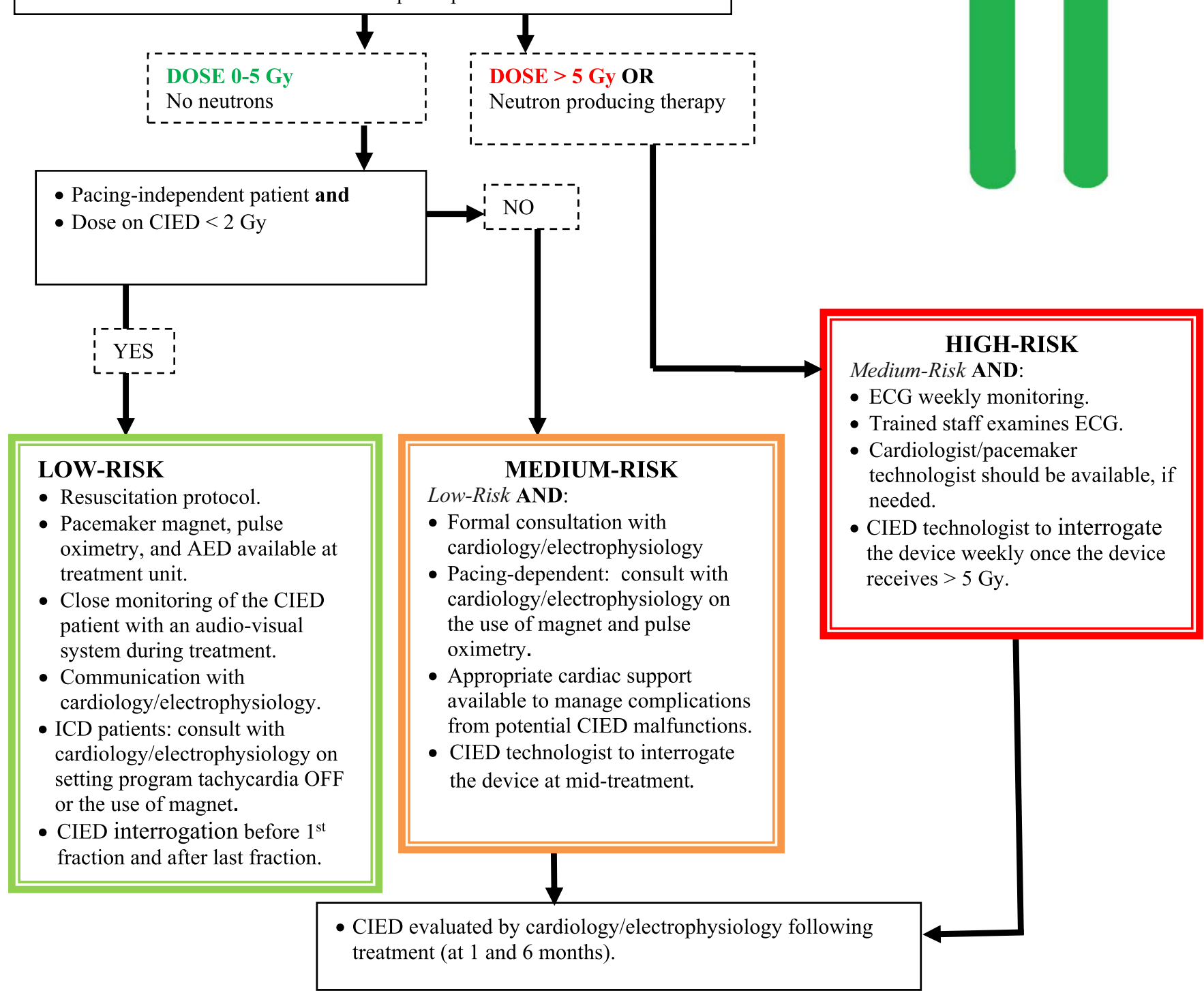

FIG. 5. Flowchart or recommended guidelines, definition of patient Risk Categories (adapted and modified from Hurkmans et al. ${ }^{159}$ ).

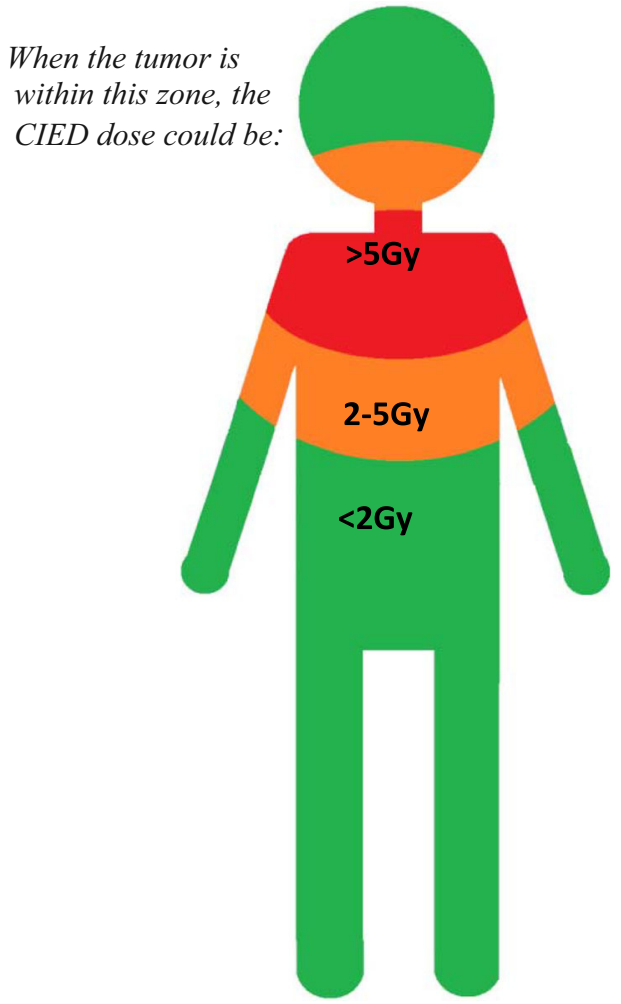

Medium-Risk AND:

ECG weekly monitoring

Trained staff examines ECG. technologist should be available, if needed.

CIED technologist to interrogate the device weekly once the device receives $>5$ Gy.

Pacing-dependent: consult with

Appropriate cardiac support available to manage complications potential CIED malfunctions.

CIED technologist to interrogate the device at mid-treatment.
Cardiologist/pacemake

CIED evaluated by cardiology/electrophysiology following treatment (at 1 and 6 months) 
representative available at the time of treatment. A referral to a cardiology clinic might be warranted in selected cases if reprogramming of the device is preferred.

It is imperative to identify patients with CIEDs as early in their RT treatment process as possible. To identify these patients, clinics should ensure that all patients that may undergo RT be asked during their initial consultation whether they have any implanted electronic devices. In addition, clinics should institute written policies for handling such patients, including consultation with the cardiology department or cardiology care provider to obtain information on the device and determine the patient's pacing dependency. These policies should include the necessary workflow for patients, clearly indicate who is responsible for each step of the process, and be periodically reviewed

\section{Management of Patients with CIED Suggested Medical Staff Responsibilities}

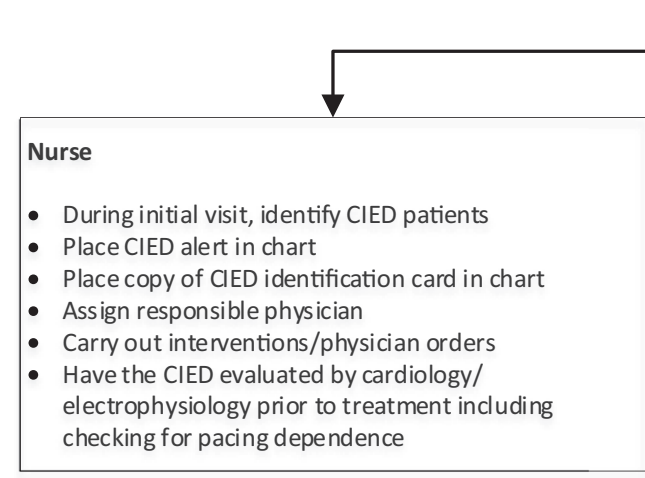

Simulation

- Exclude the CIED from the scan when the anatomy in the CIED a rea is not needed for diagnosis, target and normal tissue delineation, and radiation transport based dose calculations

- Estimate the approximate distance from the field edge to the CIED

- Record distance in chart

Dosimetrist

- If CIED is within $3 \mathrm{~cm}$ of the field edge or $5 \%$ is odose line, contour the CIED as a structure and determine $\mathrm{DVH} /$ point dose
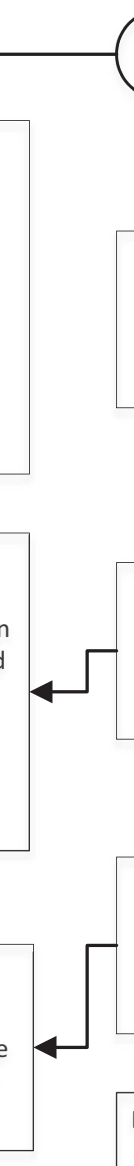

for CIED

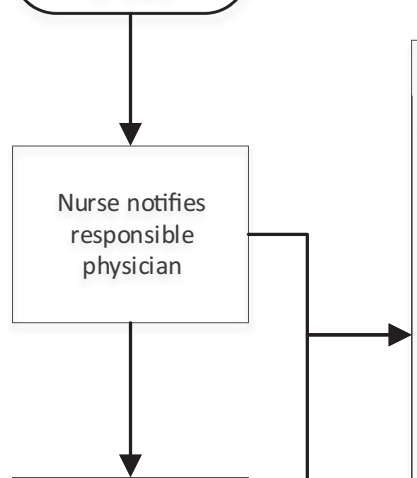

Nurse notifies simulation

Nurse notifies dosimetrist and physicist

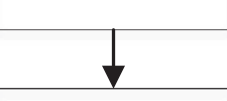

Dosimetrist notifies physician of point dose and/or distance from field edge to CIED

Therapist
- Notify nurse of patient start date for coordination
with device monitoring
- Ensure CIED guidelines are set forth as ordered by the
physician
Notify physics for placement of in vivo measurement
device on the first day of treatment

Physician

- Consult with cardiology/electrophysiology, and in conjunction, determine the risk level (low, medium, or high)

- Order in vivo measurement when appropriate

- Consult physics/dosimetry to assess dose to the CIED

- CIED should not fall directly within the radiation field

- Assess RT risk to CIED using TG203 and manufacturer guidelines

- On treatment orders will be based on risk level and cardiologist/electrophysiologist recommendations

- Follow TG203 guidelines for monitoring during treatment and place orders
Physicist

- Contact manufacturer and place manufacturer guidelines in chart

- Perform in vivo measurement per TG203 by placing device on the nearest part of the CIED to the field

- Compare in vivo measurement results to manufacturer guidelines

- Notify physician of in vivo measurement results and document in chart

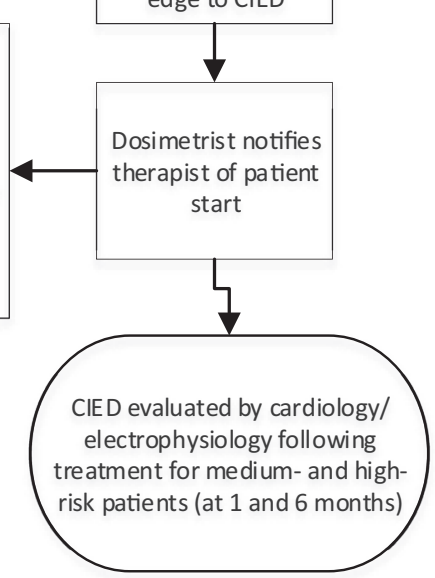

FIG. 6. Flowchart of responsibilities for management of patients with CIEDs. 
TABLE VII. Staff and departmental requirements.

\begin{tabular}{|c|c|c|c|}
\hline Department & $\begin{array}{l}\text { - Resuscitation protocol. } \\
\text { - Pacemaker magnet, pulse oximetry, and AED } \\
\text { available at treatment unit. } \\
\text { - Close monitoring of the CIED patient with an } \\
\text { audio-visual system during treatment. } \\
\text { - Communication with cardiology/ } \\
\text { electrophysiology } \\
\text { - ICD patients: consult with cardiology/ } \\
\text { electrophysiology on setting program } \\
\text { tachycardia OFF or the use of magnet. }\end{array}$ & $\begin{array}{l}\text { Low-Risk requirements } \\
\text { AND } \\
\text { - Formal consultation with cardiology/ } \\
\text { electrophysiology. } \\
\text { - Pacing dependent: consult with cardiology/ } \\
\text { electrophysiology on the use of } \\
\text { magnet and pulse oximetry. } \\
\text { - Appropriate cardiac support available to } \\
\text { manage complications from potential } \\
\text { CIED malfunctions. }\end{array}$ & $\begin{array}{l}\text { Medium-Risk requirements } \\
\text { AND } \\
\text { - ECG monitoring weekly. }\end{array}$ \\
\hline Staff & $\begin{array}{l}\text { - CIED interrogation before the first and after } \\
\text { the last treatment fractions. } \\
\text { - Radiation oncologist and MP available with } \\
\text { sufficient knowledge in the management of } \\
\text { patients with a CIED. } \\
\text { - Radiation therapists/technologists should be } \\
\text { experienced in the management of CIED } \\
\text { patients receiving radiation treatment. }\end{array}$ & $\begin{array}{l}\text { Low-Risk requirements } \\
\text { AND } \\
\text { - CIED technologist to interrogate the } \\
\text { device at mid-treatment. }\end{array}$ & $\begin{array}{l}\text { Medium-Risk requirements } \\
\text { AND } \\
\text { - Trained staff examines ECG. } \\
\text { - Cardiologist/pacemaker technologist } \\
\text { should be available, if needed. } \\
\text { - CIED technologist to interrogate the } \\
\text { device weekly once the device } \\
\text { receives }>5 \text { Gy. }\end{array}$ \\
\hline
\end{tabular}

MP: Medical Physicist.

The use of a magnet over an ICD will temporarily inhibit defibrillation therapy while it is applied. Prior to its use cardiology/electrophysiology should be consulted. Cardiology may require monitoring the patient during treatment in case defibrillation is required.

Since the use of a magnet causes the pacemaker to pace asynchronously, cardiology/electrophysiology should be consulted. Note that the magnet will cause the ICP to ignore any sensed activity and is therefore asynchronous to any intrinsic patient heart rhythm.

and updated as new information concerning the CIEDs become available. An example workflow diagram and example checklist is provided in Fig. 5 and Table VIII, respectively, for managing patients with CIEDs. It is prudent for a facility to include a section regarding potential malfunction of CIEDs due to radiation in their patient consent form.

Once patients are identified as having a CIED, their baseline cardiac function, CIED function, and battery life expectancy should be evaluated. It is advised to schedule a device interrogation with the cardiology department prior to the patient's first treatment, and preferably, prior to their treatment planning simulation if such a check has not been performed within the preceding 3 months. ${ }^{7,159}$ During the patient's cardiac consultation, the following information should be documented: CIED make, model, whether the device contains active leads, date of implantation, patient's device dependency, position of the device, purpose for the device, patient-specific device settings, and what intervention the device should deliver. ${ }^{162}$ The decision on whether a magnet should be used over the device during irradiation should also be considered in consultation with the cardiology department and be documented.

\section{B. Measures before treatment}

Prior to simulation, an MP should be consulted to evaluate the possible effects of RT on the CIED. This evaluation should consider all possible failure mechanisms. Treatment options should be considered that minimize risk to the device while not increasing morbidity to the patient. The MP should advise the radiation oncologist should take caution in selecting the imaging modality, imaging aperture size needed, as well as the beam modality, beam energy, beam dose-rate, and other treatment planning parameters for the particular patient.

The patient risk category needs to be known as soon as possible in the RT process (Table VI) as it will determine what monitoring is appropriate. Full categorization of the patient should be discussed before simulation, and should be confirmed before the first fraction. Cumulative dose to the CIED can be estimated in advance numerically (e.g., TG- $158^{132}$ ), but measurements should occur before the first fraction. It is important to realize that the accuracy of the calculation or measurement only needs to be sufficient to determine in which risk category the patient will fall. If unknown, conservative estimates should be used and the patient should be categorized in the highest risk category that might be applicable for that case.

The manufacturer should be consulted when establishing the dose and dose-rate tolerance of the CIED if possible. The recommended dose thresholds in this report are intended to provide realistic management options, especially if manufacturer thresholds are unreasonable or non-existent. If the manufacturer states a 0 Gy limit, we recommend using the guidelines in this report. If the manufacturer specifies a dose tolerance above $2 \mathrm{~Gy}$, the manufacturer tolerance can be used. The manufacturer should make available any available information about radiation and management of cardiac patients. Solan et al., ${ }^{5}$ have compiled a table of information regarding recommendations from primary manufacturers, for the management of patients with CIEDs during RT. 
TABLE VIII. RT patient management check list.

Patient Information

Date:

\section{Initial Consultation}

a. CIED alert added to patient's chart

b. Copy of CIED card made and filed in patient's chart

c. Appointment with Cardiac Electrophysiology (EP) scheduled if needed

d. Determine from cardiologist whether magnet is required

\section{Simulation stage}

a. Patient was evaluated to verify dependence on device

b. Verify CIED alert added to patient's chart

c. Verify treatment planning directive completed by physician

d. Note added to planning directive to only use photons $\leq 10 \mathrm{MV}$ and avoid physical wedges where possible

e. Contact vendor for dose threshold recommendations

\section{Planning stage}

a. Verify only $\leq 10 \mathrm{MV}$ photons used for treatment

b. Estimate dose/fraction and cumulative dose

c. Verify proximity of treatment fields to device

i. If edge of field $>10 \mathrm{~cm}$, then no further action necessary.

ii. If edge of field $<10 \mathrm{~cm}$, then continue with checklist.

d. If necessary, add note to patient's chart to place in vivo dosimeter on fraction \#1

e. Verify/adjust imaging fields do not irradiate device.

i. If device is in imaging field, use $\mathrm{kV}$ imaging where possible.

ii. If fields adjusted, add note in chart to indicate appropriate field size for imaging.

\section{First day of treatment delivery}
a. Place in vivo dosimeter on CIED at closest approach to treatment field area
b. Verify imaging field does not irradiate CIED
c. Read dosimeter and generate summary of reading for physician

5. Dose to CIED

\section{$<2$ Gy (Low-Risk)}

- Resuscitation protocol.

- Pacemaker magnet, pulse oximetry, and AED available at treatment unit.

- Close monitoring of the CIED patient with an audio-visual system during treatment.

- Communication with cardiology/electrophysiology

- ICD patients: consult with cardiology/electrophysiology on setting program tachycardia OFF or the use of magnet.

- CIED interrogation before $1^{\text {st }}$ fraction and after last fraction.

\section{2-5 Gy (Medium-Risk)}

Low-Risk requirements

$$
A N D
$$

- Formal consultation with cardiology/electrophysiology.

- Pacing-dependent: consult with

cardiology/electrophysiology on the use of magnet and pulse oximetry.

- Appropriate cardiac support available to manage complications from potential CIED malfunctions.

- CIED technologist to interrogate the device at midtreatment.
$>5$ Gy or neutrons (High-Risk Medium-Risk requirements AND

- ECG weekly monitoring.

- Trained staff examines ECG.

- Cardiologist/pacemaker technologist should be available, if needed.

- CIED technologist to interrogate the device weekly once the device receives $>5$ Gy. 
The radiation oncologist should consult with and inform the treating cardiologist about the indications for RT and, determine if the patient is pacing dependent or not. The radiation oncologist should inform the patient about the small risks involved and request the patient to report all cardiologic symptoms encountered during his/ her course of RT.

\section{C. Simulation}

Brief CT examinations have been found to be safe for patients with CIEDs. The study by Hussein et al. ${ }^{74}$ showed no strong evidence of clinical significant risks associated with direct CT irradiation of CIEDs. However, precautions may need to be taken for scans that will directly irradiate the device for longer than $3 \mathrm{~s}$ (e.g., 4DCT scans or perfusion imaging). For dynamic scans appropriate precautions may include avoiding the CIED during the CT examination or turning off the device for nondependent CIED patients when possible. Therefore, we recommend the following:

1. To minimize risk, direct CT irradiation of CIEDs should be avoided when readily possible (i.e., it should be excluded from the scan extent when the anatomy in that area is not necessary for diagnosis, target, and normal tissue delineation, radiation transport, etc.). Technologists may want to verify the location of the device with low-dose CT localization images prior to selecting the limits of the full length scan. ${ }^{15,71}$

2. CT imaging with techniques that involve helical scan with pitch $>1$ are preferred (to prevent long periods of direct irradiation of the device).

3. If it is not possible to avoid prolonged ( $>3 \mathrm{~s}$ ) direct irradiation of the device, the simulation team should carefully observe the patient for possible transient effects of the device.

4. With the introduction of magnetic resonance (MR) simulators in RT departments and the availability of MR conditional and compatible cardiac devices, such as the Medtronic Revo MRI SureScan pacing system, it is imperative to verify the CIED compatibility prior to simulating the patient. In particular, one should contact the device manufacturer to ensure the device compatibility with the magnetic field strength used during the MR simulation for planning. ${ }^{163,164}$ The HRS 2017 expert consensus report, ${ }^{22}$ which focuses on CIED management in MRI environments, provides many detailed suggestions that support and supplement these comments.

\section{D. Treatment planning}

Cardiac implantable electronic devices are composed of, and can be contoured into, many different sections. ${ }^{165}$ The electronically sensitive component of the device is the body. Dosimetric assessment should be based on the maximum dose to the part of the CIED body closest to the radiation field. Absorbed dose to the pacemaker should be evaluated by an MP and documented. 2,6,165

The CIED should not be placed in the direct radiation field if possible. ${ }^{1,6,165}$ If placement within the field is unavoidable, the physicist, radiation oncologist, and the cardiology department should engage in a consultation. The patient should be associated with the High-Risk category. However, the quality of the treatment plan should not generally be compromised for the sake of sparing the device (i.e., to minimize the relatively small risk of malfunction), because in almost all cases controlling the disease will be more important than reducing the comparatively low risk of device failure. The ultimate justification and decision may depend on the purpose of the treatment (whether curative or palliative). Regardless, the dose and risk to the device can often be minimized through appropriate treatment planning strategies, such as the use of an appropriate beam energy, gantry angles, and modality. ${ }^{2,8,166}$ The use of non-coplanar beams, ${ }^{166}$ electron beams, ${ }^{7}$ and specific targeting of the tumor (e.g., using partial breast irradiation instead of whole breast irradiation $)^{119}$ have all been used to minimize dose to the CIED. For machinespecific treatment modalities, like Cyberknife delivery, the advantage of selectively blocked entry and exit of the beams through the device areas can be employed. Consideration should be given to the possibility of repositioning (revise) the device while leads are left in place. This may be deemed an appropriate course of action, particularly if the patient has a low cardiac output and is pace dependent. However, it is not generally recommended because the potential complications, such as infections to the patient, ${ }^{159,167}$ are typically more serious than the possible risk of a device malfunction. Temporary explanation may also be an option made available by the cardiologist. The 2017 HRS expert consensus report states explicitly that moving the device is inappropriate if the device receives less than $5 \mathrm{~Gy}^{22}$ If the device is within a primary field, multiple treatment beams and/or intensity modulation segments can be utilized to block the device generator ${ }^{168}$ using the multileaf collimator. ${ }^{68}$ Although the target will not be completely covered in each segment or in the field of each beam angle, this approach is intended to minimize the dose to the CIED while covering the target within other segments or angles.

Treatment planning systems are optimized for the estimation of dose to patients. To resolve high- $Z$ components, the Hounsfield unit (HU) calibration can be enabled with an extended range HU calibration curve. ${ }^{169}$ The extended HU range should be used for in-field or near-field dosimetry. For out-of-field dosimetry, it is recommended to calculate the dose from the TPS using the normal HU range of $-1,000$ to $+1,000$ (e.g., air-to-bone), as the complexities of medium specification $\left(D_{w}\right.$ vs. $\left.D_{m}\right)$ and dose escalation from low-energy scattered x-rays have not been well documented. As treatment planning system accuracy becomes unacceptably poor, even for this type of assessment beyond $3 \mathrm{~cm}$ from the field edge, they should not be used to assess dose beyond this distance. 137 
To keep the dose to the device as low as possible, it is advisable to also keep it out of portal images or the CBCT range, if clinically reasonable.

Placing lead shielding over the device to decrease the scatter radiation (mostly head scatter) to it has been reported in literature. ${ }^{22}$ Lead shielding on the patient would not shield internally scattered radiation, nor would it meaningfully attenuate the photons leaking through the accelerator head (only a thin piece of lead could be placed on a patient). Lead shielding does reduce the superficial dose associated with electron emanating from the accelerator head. These electrons produce an elevated superficial dose (from the patient's surface to a depth of the photon beam $d_{\max }$ ). The lead shielding would block these electrons, thereby reducing the superficial dose. However, this shielding is only relevant for beams that are incident on the shielded surface of the patient (i.e., an anterior shield would have no impact on a posterior or even a lateral beam). As such, for multifield treatments, the impact of such shielding is minimal and would rarely be worth applying. Also of note, as the dose is elevated only down to $d_{\max }, 1-1.5 \mathrm{~cm}$ of bolus would shield equally as well as a piece of lead. With bolus material lighter in weight than lead, a bolus would likely be better tolerated better by the patient.

Based on the published research indicating CIED susceptibility to damage and interference under various radiations and energies, the following are recommended:

1. Determine if the patient had prior irradiation proximal to the device area.

2. Nominal $\mathrm{x}$-ray energies lower than (or equal to) $10 \mathrm{MV}$ should be used to avoid neutron production. ${ }^{55,56}$ If high photon energies (>10 MV) cannot be avoided, then the patient should be managed within the High-Risk category.

3. Irradiation with proton or neutron beams should be avoided to avoid neutron production. If used, the patient should be managed within the High-Risk category.

4. Lower dose-rates are preferred (see Section 4.C).

5. Cumulative dose should be kept at $<5$ Gy level for CIEDs (i.e., Low- to Medium-Risk categories), when possible.

6. The recommended dose thresholds of 2 and 5 Gy in this report are intended to be practical thresholds, particularly when manufacturers provide unreasonable thresholds. If the manufacturer states a 0 Gy limit, for example, we recommend using the guidelines in this report. If the manufacturer specifies a dose tolerance above $2 \mathrm{~Gy}$, that manufacturer tolerance can be used.

7. Treatment planning should include selection of the appropriate beam angles to increase the distance and shielding of the CIED. ${ }^{46}$

8. Whenever feasible, the generator for the device should be kept at least $5 \mathrm{~cm}$ from the collimated field edge (including imaging fields) to limit the dose to the device. $^{46}$
9. The use of external lead shielding is not recommended for treatment.

Prior to implementing the treatment plan, it is strongly recommended that a thorough review of the techniques used in planning process, the settings of the accelerator (e.g., MU/ min rate), and the calculated doses to target structures and the implantable device, be conducted by the MP. Where necessary, out-of-field dosimetry should be estimated and/or measured for further consideration.

\section{E. Measures during treatment}

The main points of managing a patient with CIED during RT treatment are discussed below.

1. If the CIED is more than $10 \mathrm{~cm}$ from the edge of the radiation treatment area, in vivo dosimetry is not necessary to estimate the cumulative dose to the device (which will be less than $2 \mathrm{~Gy}$ ) unless noncoplanar beams are used or there is some other condition that could unexpectedly elevate the dose to the CIED.

2. If the CIED generator is between 3 and $10 \mathrm{~cm}$ from edge of radiation treatment area, an in vivo dosimeter should be placed over the device and covered with bolus (typically $\geq 0.5 \mathrm{~cm}$ ) to measure dose from the first treatment fraction. From the measurement, the estimated cumulative dose to the CIED should be calculated and presented to the radiation oncologist. If the cumulative dose exceeds $2 \mathrm{~Gy}$, treatment planning modifications can be considered or the patient should be managed according to the Medium-Risk category. If the dose is $>5 \mathrm{~Gy}$, the patient should be managed under the High-Risk category.

3. If the nearest edge of the CIED generator is closer than $3 \mathrm{~cm}$ from edge of radiation treatment area (or within the 5\% isodose line), the treatment planning system should be used to estimate the maximum dose to the CIED. If the cumulative dose exceeds $2 \mathrm{~Gy}$, treatment planning modifications can be considered or the patient should be managed according to the MediumRisk category. If the dose is $>5 \mathrm{~Gy}$, the patient should be managed under the High-Risk category.

4. The patient, their family, and personnel working with patient should be asked (by the physician or nurse) to report any transient or prolonged cardiac symptoms. ${ }^{165}$

5. For patients with ICDs, consult with cardiology staff on the need to deactivate antitachycardia therapy temporarily during treatment sessions. Deactivation can be accomplished by placing a magnet over the ICD to inhibit tachycardia therapy, or through programming to inhibit therapy. If the ICD is programmed to inhibit, then therapy must be re-enabled following each session. A magnet will only inhibit therapy while it is in place..$^{10,46,159}$

6. For an ICP, the magnet causes the device to pace at a constant rate, where it cannot be affected by the radiation conditions. 
7. If the device is deactivated, careful patient and heart rhythm monitoring is needed. If an intracardiac electrogram does not show aberrations or morphology that would trigger antitachycardia therapy during the first fraction, then it may be concluded that the risk of an inappropriate response to delivered therapy in subsequent fractions is minimal as long as the therapy does not change. In this case, refraining from deactivating the antitachycardia function during subsequent fractions may be considered. Careful monitoring of the device by a trained staff member or the vendor representative is recommended. ${ }^{159}$

Once RT begins, patient monitoring and device interrogation should be conducted according to the patient risk category described below. Interrogation can be performed by interfacing the device with appropriate heart rhythm equipment. This can be performed by trained personnel in a physician's office, over the phone, or with self-check devices operated by the patient or a family member. ${ }^{10,162}$ The essential information obtained during interrogation is the battery life, device status, and if and when the device was activated since last interrogation. ${ }^{162}$ The reader is also referred to the 2017 HRS expert consensus report ${ }^{22}$ for further information on device interrogation.

\section{E.1. Low-Risk management}

Patients should be carefully visually and audibly monitored during each fraction. For some patients with a CIED, the cardiologist may recommend using a magnet over the device which will cause an ICP to pace at a constant rate, but will turn off an ICD. A CIED should be interrogated by a trained professional before the first fraction to establish a baseline, as well as at the completion of RT treatment.

\section{E.2. Medium-risk management}

Patients should be visually and audibly monitored during each fraction. A crash cart with external defibrillator should be at hand during all sessions. A person appropriately trained should be available within $10 \mathrm{~min}$ to help the patient in the event of a CIED malfunction. If the patient is device dependent, a cardiac specialist should be notified and be on-call for all treatment fractions (available within $10 \mathrm{~min}$ ) to address an emergent cardiac episode that may arise. ${ }^{46,159,165}$ A CIED should be interrogated before the first fraction for a baseline and then at mid-treatment and at the completion of the course of RT by a trained professional. ${ }^{159}$

\section{E.3. High-risk management}

Patient monitoring identically as for the Medium-Risk management case, plus the CIED should be interrogated after each weekly treatment. ${ }^{165}$ ECG monitoring should be conducted weekly, with the device checked within $24 \mathrm{~h}$ of the treatment by a trained professional. When a patient is at
High-Risk, because of cumulative dose (dose $>5 \mathrm{~Gy}$ ), weekly monitoring is recommended only after the device has received 5 Gy (i.e., weekly monitoring need only begin once the device receives 5 Gy). High-Risk cases associated with neutron production are at risk for all fractions and therefore High-Risk monitoring is necessary from the first fraction onward.

Depending on the risk categories of patient management, attention should also be paid to the following during and after the course of treatment:

1. If a CIED malfunction is detected during the course of RT, appropriate steps need to be taken. The patient, treating radiation oncologist, cardiologist, and responsible MP should be notified. If possible, the malfunction should be resolved (e.g., having a trained professional reprogram the device). Depending on the severity of the malfunction, it must be decided how the treatment should continue and if extra monitoring is warranted, while considering the patient's pacing dependency and number of treatment fractions remaining.

2. For Medium and High-Risk categories at the completion of RT, the device should be evaluated by a trained cardiology professional to determine whether it may need to be reprogrammed or replaced. The patient should continue to be monitored following RT at 1 and 6 months after the last RT fraction to assess possible late radiation-induced effects such as device malfunctions, functional changes, and increased sensitivity to EMI.

When treating a patient with a CIED, emergency response equipment should be readily available in case medical intervention is required (see Table VII). At a minimum, for Medium- and High-Risk category patients, this equipment should include: ${ }^{6,46,165}$

1. Crash cart - including CPR devices

2. Cardiac monitoring device, that is, ECG

3. Pacemaker magnet, pulse oximetry, and automated external defibrillator

4. Temporary transcutaneous pacing with staff trained in its use.

This equipment should be maintained by either the department of radiation oncology or cardiology. If the department or clinic does not have a crash cart and/or an Advanced Cardiac Life support-trained staff member, the clinic should have an emergency response plan in place. Following the requirements in Table VII, besides the departmental equipment requirements, the necessary staffing requirements should also be met. This includes having both a radiation oncologist and an MP with sufficient technical knowledge involved in the dose management of a CIED. If a Medium or High-Risk category patient is treated, a trained cardiology professional should be available within 10 min. 
For a patient with CIED that needs RT in an emergency setting, the risk from disease complications far exceeds the potential risk to CIED from RT. The staff should document the location of the device via photos and, in the case the device was in close proximity to the treatment field, to ensure the device was contained within the CT scan so that dose to the device is reconstructed. If the device is not in field, the patient should be able to receive a few fractions of treatment without nearing the dose thresholds and associated risk categories recommended in this report. A more detailed treatment and management plan should be produced using the recommendations in this report and in consultation with the cardiologist following the initial treatment.

\section{F. Documentation and record keeping}

During weekly chart rounds meetings, it should be known and announced whether a patient has a CIED. It should already be written in the patient's medical file. In case this is not known prior to consultation, the patient should be asked during the first visit to the RT facility. The patient (or patient's legal representative) consent should be obtained in which that person is educated and notified about the potential adverse effects of RT on CIEDs and that an ICD might be deactivated during RT. $^{19,170}$

A copy of the patient's device card should be obtained and the device manufacturer should be contacted to discuss the radiation-related issues of that device. Results of the first device interrogation should be made available to the radiation oncologist and incorporated in the patient's file. In addition, it should also be noted if the patient is pacing dependent or not, whether a magnet should be used to turn off antitachycardia therapy, and an estimate of the cumulative dose to the device. The preliminary decision to opt for device revision/explant or adaptation the RT plan, should be known before proceeding with radiation treatment delivery. This information should also be made available through the cardiology department.

It is the responsibility of the MP to calculate and measure the CIED dose for the treatment plan and note this information in the medical record. All in vivo or phantom measurements performed need to be included in the patient's file. The management strategy selected for the patient should be documented.

After RT, documentation that the cardiologist was advised to interrogate the device at 1 and 6 month post-RT to verify its functionality should be included in the patient's medical records.

\section{MANAGEMENT OF PATIENTS WITH OTHER IMPLANTABLE DEVICES}

In addition to CIEDs, there are a number of other implantable medical devices that may be seen in patients receiving RT. For example, implantable loop monitors (ILMs) are not CIEDs, but are used for cardiac diagnostics and cardiac resynchronization. These are USB-sized devices implanted under the skin to provide a continuous ECG recording of the patient's heart rhythm. Other devices include cochlear implants, ${ }^{171}$ epidural pumps, nerve stimulators, and insulin pumps. There are already a handful of case reports documenting radiation tolerance and failure. For example, cochlear implants have been shown to tolerate doses up to 80 Gy. ${ }^{172-175}$ A case report showed a Programmable Intrathecal (IT) drug delivery device receiving a dose of approximately 10 Gy from direct irradiation that did not experience any adverse effects. ${ }^{176}$ However, there is also a case report of failure of an epidural pain pump that received $28.5 \mathrm{~Gy} .{ }^{177}$

A recent study of radiation effects (6 and $18 \mathrm{MV}$ beams) on a specific manufacturer's vagus nerve stimulators (VNS) models revealed that while some models exhibited no malfunctions, others showed an apparent loss of stimulation output immediately after irradiation. The effects were dependent on beam energy and location of the device relative to the irradiation field. Cumulative dose effects were also noted (4958 Gy of tolerance dose for some models). ${ }^{178}$

However, in general, the published studies are limited in assisting with clinical management of any of these specific devices. To manage such a case, we encourage the radiation oncologist to communicate with the appropriate specialty clinic that manages the implanted device.

In general, for implanted electronic devices, specific guidance from the device manufacturer should be obtained, if available, during the planning phase of treatment. The treating physician must balance coverage of the target volume with dose delivered to the device. It is recommended that direct irradiation of the device be avoided whenever possible. The device should be tested before the start of treatment and periodically throughout treatment. Other steps may be possible as well, for example, for VNS devices, it may be possible for the generator to be programmed "off" prior to treatment, and can then be reprogrammed by the electrophysiologist after the treatment to resume stimulation to the patient's settings. Whatever the device may be, if it does fail, the dose delivered and treatment conditions (energy, dose-rate, etc.) should be documented. This information should be provided to the manufacturer. It should also be presented to the community at large (e.g., a case report), so that a status review of affects published in literature can be made possible at a later date.

In conclusion, all implantable medical devices are based on CMOS technology and have been since the late 1980s. The majority of newer medical devices entering the market are based on improved CMOS circuitry which are believed to be more radiation resistant. As manufacturers integrate this newer CMOS technology into their products, it is expected that their radiation tolerance will improve. However, it would be prudent for the MP not to simply accept that they are more radiation tolerant, but consult with the manufacturer regarding their recommended radiation dose tolerance and consider implementing the guidelines discussed in this report.

\section{SUMMARY AND CONCLUSIONS}

Management of RT patients with a CIED is essential. Safe medical practice requires that the risk to patients be reduced as much as possible. This includes minimizing the risk 
associated with CIED malfunctions. It is to this end that our task group has devised recommendations for the management of patients from simulation to postirradiation follow-up with a special emphasis on reducing potential damage to CIEDs. It is essential that this risk of CIED malfunction be weighed against other medical risks the patient may be subjected to. Such risks may include infection associated with moving/explanting the device, or even the risk of treatment failure associated with compromising the radiotherapy treatment. In principle, it is unreasonable to compromise the quality of radiotherapy and increase the risk of recurrence for the sake of reducing the risk to a CIED (which is comparatively low). Rather, the risk to the patient should be minimized by ensuring they have optimal care, including (a) minimizing risk to the CIED to the extent reasonable, and (b) appropriate monitoring of the CIED, so that device malfunctions are identified and can be resolved. This section summarizes some of the important recommendations of the task group. For further details on each recommendation, the relevant section is listed in the parentheses at the end of each bullet.

1. Management of patients should be based on device risk levels shown in Table VI (Section 7).

2. CT irradiation of CIED for longer than $3 \mathrm{~s}$ should be avoided, whenever possible (Section 8.C).

3. CIED compatibility with MRI should be verified prior to MR simulation, and protocols for safe MRI scanning should be developed and implemented (Sections 5.A.3 and 8.C)

4. If $>10 \mathrm{MV}$ photon, proton, or neutron beams are used, the patient should be managed in the High-Risk category (Section 4.B).

5. Lower dose-rates are preferred (Section 4.C).

6. The generator for the device should be kept at least $5 \mathrm{~cm}$ from the collimated field edge if possible, including imaging fields, by selection of the appropriate beam angles that increase the distance between the field edge and the CIED (Section 8.D).

7. Perform in vivo dosimetry for the first fraction if the device is $<10 \mathrm{~cm}$ from treatment area. The treatment planning system should be used in lieu of a measurement if the device is within $3 \mathrm{~cm}$ (laterally) of the field edge or 5\% isodose line for IMRT (Sections 6.C.3 and 8.E).

8. Lead should not be used for shielding CIED during treatment (Section 8.D).

9. Cumulative dose should be kept at $<5$ Gy level for CIEDs (i.e., Low- to Medium-Risk categories), as much as possible or below the manufacturer's recommended dose threshold if higher (Sections 4.A. and 7).

10. Patients should be monitored with specified frequencies and techniques (Section 8.E)

The creation of a management program for RT patients with CIED is a difficult task. This task group provides a riskbased approach for patient management along with the description of many of the issues, which must be considered before, during, and after treatment. Critical recommendations of this task group is that neutron-producing treatments be avoided, and that the cumulative dose to the CIED should be kept below 2 and 5 Gy as much as possible for pacing-dependent and pacing-independent patients, respectively. As CIEDs and RT technologies evolve, the management of patients with CIEDs should also evolve. Imaging and treatment delivery machine evolution may enable a more safe utilization of techniques when a CIED remains implanted. This task group concludes that the appropriate implementation of the recommendations provided in this report will offer clinics the needed tools to develop a safer and effective treatment management program for patients with CIEDs.

\footnotetext{
${ }^{\dagger}$ The report was endorsed by the European Society for Radiotherapy and Oncology (ESTRO)-Advisory Committee on Radiation Oncology Practice (ACROP).

${ }^{*}$ The co-chairs of TG-203 are first co-authors on the report. They contributed equally to the report.

a) Authors to whom correspondence should be addressed. Electronic mails: Moyed.Miften@ucdenver.edu and Dimitris.Mihailidis@pennmedicine. upenn.edu.
}

\section{REFERENCES}

1. Marbach JR, Sontag MR, Van Dyk J, Wolbarst AB. Management of radiation oncology patients with implanted cardiac pacemakers: report of AAPM Task Group No. 34. American Association of Physicists in Medicine. Med Phys. 1994;21:85-90.

2. Last A. Radiotherapy in patients with cardiac. Br J Radiol. 1998;71: 4-10.

3. Niehaus M, Tebbenjohanns J. Electromagnetic interference in patients with implanted pacemakers or cardioverter-defibrillators. Heart. 2001; 86:246-248.

4. Mouton J, Haug R, Bridier A, Dodinot B, Eschwege F. Influence of high-energy photon beam irradiation on pacemaker operation. Phys Med Biol. 2002;47:2879-2893.

5. Solan A, Solan M, Bednarz G, Goodkin M. Treatment of patients with cardiac pacemakers and implantable cardioverter-defibrillators during radiotherapy. Int J Radiat Oncol Biol Phys. 2004;59:897-904.

6. Hurkmans CW, Scheepers E, Springorum BGF, Uiterwaal H. Influence of radiotherapy on the latest generation of implantable cardioverter-defibrillators. Int J Radiat Oncol Biol Phys. 2005;63: 282-289.

7. Sundar S, Symonds R, Deehan C. Radiotherapy to patients with artificial cardiac pacemakers. Cancer Treat Rev. 2005;31:474-486.

8. Gossman MS, Graves-Calhoun AR, Wilkinson JD. Establishing radiation therapy treatment planning effects involving implantable pacemakers and implantable cardioverter-defibrillators. J Appl Clin Med Phys. 2010;11:33-45.

9. Oshiro Y, Sugahara S, Noma M, et al. Proton beam therapy interference with implanted cardiac pacemakers. Int J Radiat Oncol Biol Phys. 2008;72:723-727.

10. Gelblum DY, Amols H. Implanted cardiac defibrillator care in radiation oncology patient population. Int J Radiat Oncol Biol Phys. 2009; 73:1525-1531.

11. Bradley PD, Normand E. Single event upsets in implantable cardioverter defibrillators. Nucl Sci IEEE Trans. 1998;45:2929-2940.

12. Raitt MH, Stelzer KJ, Laramore GE, et al. Runaway pacemaker during high-energy neutron radiation therapy. Chest. 1994;106:955-957.

13. McCollough $\mathrm{CH}$, Zhang J, Primak AN, Clement WJ, Buysman JR. Effects of CT irradiation on implantable cardiac rhythm management devices. Radiology. 2007;243:766-774

14. Yamaji S, Imai S, Saito F, Yagi H, Kushiro T, Uchiyama T. Does highpower computed tomography scanning equipment affect the operation of pacemakers?. Circ J. 2006;70:190-197. 
15. ECRI organization. CT scans can affect the operation of implanted electronic devices. Health Devices. 2007;36:136-138.

16. Martin ET, Coman JA, Shellock FG, Pulling CC, Fair R, Jenkins K. Magnetic resonance imaging and cardiac pacemaker safety at 1.5-Tesla. J Am Coll Cardiol. 2004;43:1315-1324.

17. Zweng A, Schuster R, Hawlicek R, Weber HS. Life-threatening pacemaker dysfunction associated with therapeutic radiation: a case report. Angiology. 2008;60:509-512.

18. Kobayashi H, Shiraishi K, Tsuchiya H, et al. Soft errors in SRAM devices induced by high energy neutrons, thermal neutrons and alpha particles. Electron Devices Meeting, IEDM '02. 2002.

19. Lester JF, Evans LM, Yousef Z, Penney A, Brown PN, Perks R. A national audit of current cardiac device policies from radiotherapy centres across the UK. Clin Oncol. 2014;26:45-50.

20. Soejima T, Yoden E, Nishimura Y, et al. Radiation therapy in patients with implanted cardiac pacemakers and implantable cardioverter defibrillators: a prospective survey in Japan. J Radiat Res. 2011;52: $516-521$.

21. Gossman MS, Wilkinson JD, Mallick A. Treatment approach, delivery, and follow-up evaluation for cardiac rhythm disease management patients receiving radiation therapy: retrospective physician surveys including chart reviews at numerous centers. Med Dosim. 2014;39: 320-324.

22. Indik JH, Gimbel JR, Abe H, et al. HRS expert consensus statement on magnetic resonance imaging and radiation exposure in patients with cardiovascular implantable electronic devices. Heart Rhythm. 2017;14: e97-e153.

23. Kerkmeijer LGW, Fuller CD, Verkooijen HM, et al. The MRI-linear accelerator consortium: evidence-based clinical introduction of an innovation in radiation oncology connecting researchers, methodology, data collection, quality assurance, and technical development. Front Oncol. 2016;6:1-6.

24. Myers D. What happens to semiconductors in a nuclear environment. Electronics. 1978;51:131-133.

25. Katzenberg CA, Marcus FI, Heusinkveld RS, Mammana RB. Pacemaker failure due to radiation therapy. Pacing Clin Electrophysiol. 1982;5:156-159.

26. Pourhamidi AH. Radiation effect on implanted pacemakers. Chest. 1983;84:499-500.

27. Quertermous T, Megahy MS, Das Gupta DS, Griem ML. Pacemaker failure resulting from radiation damage. Radiology. 1983;148: $257-258$.

28. Lewin AA, Serago CF, Schwade JG, Abitbol AA, Margolis SC. Radiation induced failures of complementary metal oxide semiconductor containing pacemakers: a potentially lethal complication. Int $J$ Radiat Oncol Biol Phys. 1984;10:1967-1969.

29. Elders J, Kunze-Busch M, Jan Smeenk R, Smeets JLRM. High incidence of implantable cardioverter defibrillator malfunctions during radiation therapy: neutrons as a probable cause of soft errors. Europace. 2013;15:60-65.

30. Maxted KJ. The effect of therapeutic x-radiation on a sample of pacemaker generators. Phys Med Biol. 1984;29:1143-1146.

31. Venselaar JL. The effects of ionizing radiation on eight cardiac pacemakers and the influence of electromagnetic interference from two linear accelerators. Radiother Oncol. 1985;3:81-87.

32. Venselaar JL, Van Kerkoerle HL, Vet AJ. Radiation damage to pacemakers from radiotherapy. Pacing Clin Electrophysiol. 1987;10:538542.

33. Rodriguez F, Filimonov A, Henning A, Coughlin C, Greenberg M. Radiation-induced effects in multiprogrammable pacemakers and implantable defibrillators. Pacing Clin Electrophysiol. 1991;14: 2143-2153.

34. Zaremba T, Jakobsen AR, Thøgersen AM, Oddershede L, Riahi S. The effect of radiotherapy beam energy on modern cardiac devices: an in vitro study. Europace. 2013;16:612-616.

35. Hauser RG, Hayes DL, Epstein AE, et al. Multicenter experience with failed and recalled implantable cardioverter-defibrillator pulse generators. Heart Rhythm. 2006;3:640-644.

36. Lelakowski J, Majewski J, Bednarek J, Malecka B, Zabek A. Pacemaker dependency after pacemaker implantation. Cardiology Journal. 2007; 14:83-86.
37. Korantzopoulos P, Letsas KP, Grekas G, Goudevenos JA. Pacemaker dependency after implantation of electrophysiological devices. Europace. 2009;11:1151-1155.

38. Levin PA. Letter to the Editor-Pacemaker dependency after pacemaker implantation. Cardiol J. 2007;14:318-320.

39. Calfee RV. Therapeutic radiation and pacemakers. Pacing Clin Electrophysiol. 1982; 5:160-161.

40. Ma TP, Dressendorfer PV. Ionizing Radiation Effects in MOS Devices and Circuits. New York, NY: Wiley; 1989.

41. Little FA. Pacemakers in radiotherapy. Clin Oncol. 1994;6:211-212.

42. Souliman SK, Christie J. Pacemaker failure induced by radiotherapy. Pacing Clin Electrophysiol. 1994;17:270-273.

43. Gomez DR, Poenisch F, Pinnix CC, et al. Malfunctions of implantable cardiac devices in patients receiving proton beam therapy: incidence and predictors. Int J Radiat Oncol Biol Phys. 2013;87:570-575.

44. Brenner DJ, Hall EJ. Secondary neutrons in clinical proton radiotherapy: a charged issue. Radiother Oncol. 2008;86:165-170.

45. Brooks C, Mutter M. Pacemaker failure associated with therapeutic radiation. Am J Emerg Med. 1988;6:591-593.

46. Hudson F, Coulshed D, D'Souza E, Baker C. Effect of radiation therapy on the latest generation of pacemakers and implantable cardioverter defibrillators: a systematic review. J Med Imaging Radiat Oncol. 2010;54:53-61.

47. Mollerus M, Naslund L, Lipinski M, Meyer A, Libey B, Dornfeld K. Radiation tolerance of contemporary implantable cardioverter-defibrillators. J Interv Card Electrophysiol. 2014;39:171-175.

48. Hurkmans CW, Scheepers E, Springorum BGF, Uiterwaal H. Influence of radiotherapy on the latest generation of pacemakers. Radiother Oncol. 2005;76:93-98.

49. Uiterwaal H, Springorum BG, Scheepers E, de Ruiter GS, Hurkmans CW. Interference detection in implantable defibrillators induced by therapeutic radiation therapy. Neth Heart J. 2006;14: 330-334.

50. Kry SF, Salehpour M, Titt U, White RA, Stovall M, Monte Followill D. Carlo study shows no significant difference in second cancer risk between 6- and 18-MV intensity-modulated radiation therapy. Radiother Oncol. 2009;91:132-137.

51. Fontenot J, Taddei P, Zheng Y, Mirkovic D, Jordan T, Newhauser W. Equivalent dose and effective dose from stray radiation during passively scattered proton radiotherapy for prostate cancer. Phys Med Biol. 2008;53:1677-1688.

52. Tondato F, Ng DW, Srivathsan K, Altemose GT, Halyard MY, Scott LR. Radiotherapy-induced pacemaker and implantable cardioverter defibrillator malfunction [published online ahead of print 2009/05/08]. Expert Rev Med Devices. 2009;6:243-249.

53. Koivunoro H, Seren T, Hyvonen H, et al. Epithermal neutron beam interference with cardiac pacemakers. Appl Radiat Isot. 2011;69: 1904-1906.

54. Hurkmans C, Schmeets I, Uiterwaal H. In regard to Solan et al.: Treatment of patients with cardiac pacemakers and implantable cardioverter-defibrillators during radiotherapy (Int J Radiat Oncol Biol Phys 2004;59:897-904). Int J Radiat Oncol Biol Phys. 2004;60: 1662-1663.

55. Franco L, Gomez F, Iglesias A, et al.SEUs on commercial SRAM induced by low energy neutrons produced at a clinical linac facility. European Congress RADECS 2005Cap d'Agde, France; 2005.

56. Wilkinson JD, Bounds C, Brown T, Gerbi BJ, Peltier J. Cancer-radiotherapy equipment as a cause of soft errors in electronic equipment. Device Mater Reliab IEEE Trans. 2005;5:449-451.

57. Grant JD, Jensen GL, Tang C, et al. Radiotherapy-induced malfunction in contemporary cardiovascular implantable electronic devices: Clinical incidence and predictors. JAMA Oncol. 2015;1:624-632.

58. Johnston AH, Hughlock BW, Baze MP, Plaag RE. The effect of temperature on single-particle latchup. Nuc Sci IEEE Trans. 1991;38:14351441.

59. Howell RM, Kry SF, Burgett E, Hertel NE, Followill DS. Secondary neutron spectra from modern Varian, Siemens, and Elekta linacs with multileaf collimators. Med Phys. 2009;36:4027-4038.

60. Followill DS, Stovall MS, Kry SF, Ibbott GS. Neutron source strength measurements for Varian, Siemens, Elekta, and General Electric linear accelerators. J Appl Clin Med Phys. 2003;4:189-194. 
61. Sánchez-Doblado F, Domingo C, Gómez F, et al. Estimation of neutron-equivalent dose in organs of patients undergoing radiotherapy by the use of a novel online digital detector. Phys Med Biol. 2012;57:6167.

62. Reft CS, Runkel-Muller R, Myrianthopoulos L. In vivo and phantom measurements of the secondary photon and neutron doses for prostate patients undergoing 18 MV IMRT. Med Phys. 2006;33:3734-3742.

63. Kry SF, Howell RM, Salehpour M, Followill DS. Neutron spectra and dose equivalents calculated in tissue for high-energy radiation therapy. Med Phys. 2009;36:1244

64. Lin J-P, Chu T-C, Lin S-Y, Liu M-T. The measurement of photoneutrons in the vicinity of a Siemens Primus linear accelerator. Appl Radiat Isot. 2001;55:315-321.

65. Cardenas CE, Nitsch PL, Kudchadker RJ, Howell RM, Kry SF. Out-offield doses and neutron dose equivalents for electron beams from modern Varian and Elekta linear accelerators. J Appl Clin Med Phys. 2016;17:442-455.

66. Xu XG, Bednarz B, Paganetti H. A review of dosimetry studies on external-beam radiation treatment with respect to second cancer induction. Phys Med Biol. 2008;53:R193-R241.

67. Zheng Y, Newhauser W, Fontenot J, Taddei P, Mohan R. Monte Carlo study of neutron dose equivalent during passive scattering proton therapy. Phys Med Biol. 2007;52:4481-4496.

68. Ferrara T, Baiotto B, Malinverni G, et al. Irradiation of pacemakers and cardio-defibrillators in patients submitted to radiotherapy: a clinical experience. Tumori. 2010;96:76-83.

69. Stovall M, Blackwell CR, Cundiff J, et al. Fetal dose from radiotherapy with photon beams: report of AAPM Radiation Therapy Committee Task Group No. 36. Med Phys. 1995;22:63-82.

70. Prisciandaro JI, Makkar A, Fox CJ, et al. Dosimetric review of cardiac implantable electronic device patients receiving radiotherapy. J Appl Clin Med Phys. 2015;16:254-263.

71. FDA Preliminary Public Health Notification: Possible Malfunction of Electronic Medical Devices Caused by Computed Tomography (CT) Scanning. Washington, D.C.: U.S. Food and Drug Administration; 2008:1.

72. Kirova YM, Menard J, Chargari C, Mazal A, Kirov K. Case study thoracic radiotherapy in an elderly patient with pacemaker: the issue of pacing leads. Med Dosim. 2012;37:192-194.

73. Hayes DL, Wang PJ, Reynolds DW, et al. Interference with cardiac pacemakers by cellular telephones. New Engl J Med. 1997;336:1473-1479.

74. Hussein A, Abutaleb A, Jeudy J, et al. Safety of computed tomography in patients with cardiac rhythm management devices. $J$ Am Coll Cardiol. 2014;63:1769-1775.

75. Diederich S, Lenzen H. Radiation exposure associated with imaging of the chest: comparison of different radiographic and computed tomography techniques. Cancer. 2000;89:2457-2460.

76. Kan MW, Leung LH, Wong W, Lam N. Radiation dose from cone beam computed tomography for image-guided radiation therapy. Int $J$ Radiat Oncol Biol. 2008;70:272-279.

77. Keall PJ, Starkschall G, Shukla H, et al. Acquiring 4D thoracic CT scans using a multislice helical method. Phys Med Biol. 2004;49:20532067.

78. Moore CJ, Amer A, Marchant T, et al. Developments in and experience of kilovoltage X-ray cone beam image-guided radiotherapy. $\mathrm{BrJ}$ Radiol. 2006;79:S66-S78.

79. Murphy MJ. Tracking moving organs in real time. Semin Radiat Oncol. 2004;14:91-100.

80. Ding GX, Alaei P, Curran B, et al. Image guidance doses delivered during radiotherapy: quantification, management, and reduction: report of the AAPM Therapy Physics Committee Task Group 180. Med Phys. 2018;45:e84-e99.

81. Murphy MJ, Balter J, Balter S, et al. The management of imaging dose during image-guided radiotherapy: report of the AAPM Task Group 75. Med Phys. 2007;34:4041-4063.

82. Endo M, Nishizawa K, Iwai K, et al. Image characteristics and effective dose estimation of a cone beam CT using a video-fluoroscopic system. Nucl Sci IEEE Trans. 1999;46:686-690.

83. Faddegon BA, Wu V, Pouliot J, Gangadharan B, Bani-Hashemi A. Low dose megavoltage cone beam computed tomography with an unflattened 4 MV beam from a carbon target. Med Phys. 2008;35:57775786.
84. Gayou O, Parda DS, Johnson M, Miften M. Patient dose and image quality from mega-voltage cone beam computed tomography imaging. Med Phys. 2007;34:499-506.

85. Morin O, Gillis A, Descovich M, et al. Patient dose considerations for routine megavoltage cone-beam CT imaging. Med Phys. 2007;34: $1819-1827$.

86. Miften M, Gayou O, Reitz B, Fuhrer R, Leicher B, Parda DS. IMRT planning and delivery incorporating daily dose from mega-voltage cone-beam computed tomography imaging. Med Phys. 2007;34: 3760-3767.

87. Flynn RT, Hartmann J, Bani-Hashemi A, et al. Dosimetric characterization and application of an imaging beam line with a carbon electron target for megavoltage cone beam computed tomography. Med Phys. 2009:36:2181-2192.

88. Meeks SL, Harmon JF Jr, Langen KM, Willoughby TR, Wagner TH, Kupelian PA. Performance characterization of megavoltage computed tomography imaging on a helical tomotherapy unit. Med Phys. 2005;32:2673-2681.

89. Baikoussis NG, Apostolakis E, Papakonstantinou NA, Sarantitis I, Dougenis D. Safety of magnetic resonance imaging in patients with implanted cardiac prostheses and metallic cardiovascular electronic devices. The Annals of thoracic surgery. 2011;91:20062011.

90. Cohen JD, Costa HS, Russo RJ. Determining the risks of magnetic resonance imaging at 1.5 tesla for patients with pacemakers and implantable cardioverter defibrillators. Am J Cardiol. 2012;110:16311636.

91. Zikria JF, Machnicki S, Rhim E, Bhatti T, Graham RE. MRI of patients with cardiac pacemakers: a review of the medical literature. Am J Roentgenol. 2011;196:390-401.

92. Levine GN, Gomes AS, Arai AE, et al. Safety of magnetic resonance imaging in patients with cardiovascular devices. Circulation. 2007; 116:2878-2891.

93. Jung W, Zvereva V, Hajredini B, Jäckle S. Safe magnetic resonance image scanning of the pacemaker patient: current technologies and future directions. Europace. 2012;14:631-637.

94. Abner AL, Meskell P, Carrozza J, Boiselle P, Holupka EJ. Dose to cardiac pacemakers and implanted defibrillators from beta and gamma coronary artery brachytherapy. Int $J$ Radiat Oncol Biol Phys. 2002:54:301.

95. Steidley KD, Steidley DE. Pacemaker/ICD Irradiation Policies in Radiation Oncology. In https://www.ipen.br/biblioteca/cd/irpa/2004/files/4e 26.pdf. 2004:1-7.

96. Croshaw R, Lappinen E, Julian T, Trombetta M. Avoiding mastectomy: accelerated partial breast irradiation for breast cancer patients with pacemakers or defibrillators. Ann Surg Oncol. 2011;18:35003505.

97. Jacob D, Chen H, Simpson L. SU-E-T-596: high dose brachytherapy planning of a left breast cancer patient with in situ pacemaker. Med Phys. 2011;38:3626.

98. Kim Y, Arshoun Y, Trombetta MG. Pacemaker/implantable cardioverter-defibrillator dose in balloon high-dose-rate brachytherapy for breast cancer treatment. Brachytherapy. 2012;11:380-386.

99. Sung W, Kim S, Kim J-i, et al. Dosimetric perturbations due to an implanted cardiac pacemaker in MammoSite ${ }^{\circledR}$ treatment. Med Phys. 2012;39:6185-6191.

100. Keshtgar MR, Eaton DJ, Reynolds C, et al. Pacemaker and radiotherapy in breast cancer: is targeted intraoperative radiotherapy the answer in this setting? Radiat Oncol. 2012;7:1-4.

101. Kase KR, Svensson GK. Head scatter data for several linear accelerators (4-18 MV). Med Phys. 1986;13:530-532.

102. Vanhavere F, Huyskens D, Struelens L. Peripheral neutron and gamma doses in radiotherapy with an $18 \mathrm{MV}$ linear accelerator. Radiat Prot Dosim. 2004;110:607-612.

103. Kry SF, Titt U, Pönisch F, et al. A Monte Carlo model for calculating out-of-field dose from a Varian 6MV beam. Med Phys. 2006;33:44054413 .

104. Howell RM, Hertel NE, Wang Z, Hutchinson J, Fullerton GD. Calculation of effective dose from measurements of secondary neutron spectra and scattered photon dose from dynamic MLC IMRT for $6 \mathrm{MV}, 15 \mathrm{MV}$, and 18MV beam energies. Med Phys. 2006;33:360-368. 
105. Kry SF, Titt U, Followill D, et al. A Monte Carlo model for out-of-field dose calculation from high-energy photon therapy. Med Phys. 2007;34:3489-3499.

106. Starkschall G, George FJS, Zellmer D. Surface dose for megavoltage photon beams outside the treatment field. Med Phys. 1983;10:906-910.

107. Mao X, Kase K, Liu J, Nelson W, Kleck J, Johnsen S. Neutron sources in the Varian Clinac $2100 \mathrm{C} / 2300 \mathrm{C}$ medical accelerator calculated by the EGS4 code. Health Phys. 2100C;72:524-529.

108. Hashii H, Hashimoto T, Okawa A, et al. Comparison of the effects of high-energy photon beam irradiation (10 and $18 \mathrm{MV}$ ) on 2 types of implantable cardioverter-defibrillators. Int J Radiat Oncol Biol Phys. 2013;85:840-845.

109. d'Errico F, Luszik-Bhadra M, Nath R, Siebert BRL, Wolf U. Depth dose-equivalent and effective energies of photoneutrons generated by 6-18 MV X-ray beams for radiotherapy. Health Phys. 2001; 80:4-11.

110. Mesoloras G, Sandison GA, Stewart RD, Farr JB, Hsi WC. Neutron scattered dose equivalent to a fetus from proton radiotherapy of the mother. Med Phys. 2006;33:2479-2490.

111. Kry SF, Johnson JL, White RA, Howell RM, Kudchadker RJ, Gillin MT. Neutron-induced electronic failures around a high-energy linear accelerator. Med Phys. 2011;38:34-39.

112. Hoecht S, Rosenthal P, Sancar D, et al. Implantable cardiac defibrillators may be damaged by radiation therapy. J Clin Oncol. 2002;20:2212-2213.

113. Thomas D, Becker R, Katus HA, Schoels W, Karle CA. Radiation therapy-induced electrical reset of an implantable cardioverter defibrillator device located outside the irradiation field. $J$ Electrocardiol. 2004:37:73-74.

114. Nemec J. Runaway implantable defibrillator-a rare complication of radiation therapy. Pacing Clin Electrophysiol. 2007;30:716-718.

115. Nibhanupudy JR, de Jesus MA, Fujita M, Goldson AL. Radiation dose monitoring in a breast cancer patient with a pacemaker: a case report. $J$ Natl Med Assoc. 2001;93:278-281.

116. John J, Kaye GC. Shock coil failure secondary to external irradiation in a patient with implantable cardioverter defibrillator. Pacing Clin Electrophysiol. 2004;27:690-691.

117. Sepe S, Schaffer P, Krimmel K, Schaffer M. Irradiation treatment of laryngeal cancer in a patient with an implantable cardioverter-defibrillator (ICD). Onkologie. 2007;30:378-380.

118. Lau DH, Wilson L, Stiles MK, et al. Defibrillator reset by radiotherapy. Int J Cardiol. 2008;130:e37-e38.

119. Munshi A, Wadasadawala T, Kumar Sharma P, et al. Radiation therapy planning of a breast cancer patient with in situ pacemaker-challenges and lessons. Acta Oncol. 2008;47:255-260.

120. DiBiase S, Eagen J, Dufendach C. The influence of intensity modulated radiation therapy (IMRT) on cardiac devices in patients undergoing treatment for prostate cancer. Int J Radiat Oncol Biol Phys. 2011;81:2

121. Dasgupta T, Barani IJ, Roach M. Successful radiation treatment of anaplastic thyroid carcinoma metastatic to the right cardiac atrium and ventricle in a pacemaker-dependent patient. Radiat Oncol. 2011;6:16.

122. Tsekos A, Momm F, Brunner M, Guttenberger R. The cardiac pacemaker patient-might the pacer be directly irradiated?. Acta Oncol. 2000;39:881-883.

123. Kapa S, Fong L, Blackwell CR, Herman MG, Schomberg PJ, Hayes DL. Effects of scatter radiation on ICD and CRT function. Pacing Clin Electrophysiol. 2008;31:727-732.

124. Antolak JA, Strom EA. Fetal dose estimates for electron-beam treatment to the chest wall of a pregnant patient. Med Phys. 1998;25:23882391.

125. Chow JC, Grigorov GN. Peripheral dose outside applicators in electron beams. Phys Med Biol. 2006;51:N231-N240.

126. Yeboah C, Karotki A, Hunt D, Holly R. Quantification and reduction of peripheral dose from leakage radiation on Siemens primus accelerators in electron therapy mode. J Appl Clin Med Phys. 2010;11:154-172.

127. Kaderka R, Schardt D, Durante M, et al. Out-of-field dose measurements in a water phantom using different radiotherapy modalities. Phys Med Biol. 2012;57:5059-2074.
128. Hashimoto T, Isobe $\mathrm{T}$, Hashii $\mathrm{H}$, et al. Influence of secondary neutrons induced by proton radiotherapy for cancer patients with implantable cardioverter defibrillators. Radiat Oncol. 2012;7:10.

129. Clasie B, Wroe A, Kooy H, et al. Assessment of out-of-field absorbed dose and equivalent dose in proton fields. Med Phys. 2010;37:311-321.

130. Jarlskog CZ, Lee C, Bolch WE, Xu XG, Paganetti H. Assessment of organ-specific neutron equivalent doses in proton therapy using computational whole-body age-dependent voxel phantoms. Phys Med Biol. 2008;53:693-717.

131. Schneider U, Agosteo S, Pedroni E, Besserer J. Secondary neutron dose during proton therapy using spot scanning. Int J Radiat Oncol Biol Phys. 2002;53:244-251.

132. Kry SF, Bednarz B, Howell RM, et al. AAPM TG 158: measurement and calculation of doses outside the treated volume from external-beam radiation therapy. Med Phys. 2017;44:e391-e429.

133. Scarboro SB, Followill DS, Howell RM, Kry SF. Variations in photon energy spectra of a $6 \mathrm{MV}$ beam and their impact on TLD response. Med Phys. 2011;38:2619-2628.

134. Scarboro SB, Followill DS, Kerns JR, White RA, Kry SF. Energy response of optically stimulated luminescent dosimeters for non-reference measurement locations in a $6 \mathrm{MV}$ photon beam. Phys Med Biol. 2012;57:2505-2515.

135. Mobit P, Agyingi E, Sandison G. Comparison of the energy-response factor of $\mathrm{LiF}$ and $\mathrm{Al} 2 \mathrm{O} 3$ in radiotherapy beams. Radiat Prot Dosimetry. 2006;119:497-499.

136. Reft CS. The energy dependence and dose response of a commercial optically stimulated luminescent detector for kilovoltage photon, megavoltage photon, and electron, proton, and carbon beams. Med Phys. 2009;36:1690-1699.

137. Howell RM, Scarboro SB, Kry SF, Yaldo DZ. Accuracy of out-of-field dose calculations by a commercial treatment planning system. Phys Med Biol. 2010;55:6999-7008.

138. Huang JY, Followill DS, Wang XA, Kry SF. Accuracy and sources of error of out-of field dose calculations by a commercial treatment planning system for intensity-modulated radiation therapy treatments. $J$ Appl Clin Med Phys. 2013;14:186-197.

139. Joosten A, Matzinger O, Jeanneret-Sozzi W, Bochud F, Moeckli R. Evaluation of organ-specific peripheral doses after 2-dimensional, 3-dimensional and hybrid intensity modulated radiation therapy for breast cancer based on Monte Carlo and convolution/superposition algorithms: Implications for secondary cancer risk assessment. Radiother Oncol. 2013;106:33-41.

140. van der Giessen PH. Peridose, a software program to calculate the dose outside the primary beam in radiation therapy. Radiother Oncol. 2001;58:209-213.

141. Kry SF, Price M, Followill D, Mourtada F, Salehpour M. The use of LiF (TLD-100) as an out-of-field dosimeter. J Appl Clin Med Phys. 2007;8:169-175.

142. Kry SF, Starkschall G, Antolak JA, Salehpour M. Evaluation of the accuracy of fetal dose estimates using TG-36 data. Med Phys. 2007;34:1193.

143. Bednarz B, Xu XG. A feasibility study to calculate unshielded fetal doses to pregnant patients in 6-MV photon treatments using Monte Carlo methods and anatomically realistic phantoms. Med Phys. 2008;35:3054-3061.

144. Bednarz B, Monte XuXG. Carlo modeling of a 6 and 18 MV Varian Clinac medical accelerator for in-field and out-of-field dose calculations: development and validation. Phys Med Biol. 2009;54:N43N47.

145. Edwards CR, Mountford PJ. Near surface photon energy spectra outside a 6 MV field edge. Phys Med Biol. 2004;49:N293-N301.

146. Chan MF, Song Y, Dauer LT, Li J, Huang D, Burman C. Estimating dose to implantable cardioverter-defibrillator outside the treatment fields using a skin QED diode, optically stimulated luminescent dosimeters, and LiF thermoluminescent dosimeters. Med Dosim. 2012;37:334-338.

147. Snow JR, Micka JA, DeWerd LA. Microionization chamber air-kerma calibration coefficients as a function of photon energy for x-ray spectra in the range of $20-250 \mathrm{kVp}$ relative to 60Co. Med Phys. 2013;40:041711. 
148. Kry SF, Alvarez P, Cygler JE, et al. AAPM TG 191 Clinical Use of Luminescent Dosimeters: TLDs and OSLDs. Med Phys. 2019. https:// doi.org/10.1002/mp.13839.

149. Mendez R, Iniguez MP, Barquero R, et al. Response components of $\mathrm{LiF}$ : Mg, Ti around a moderated Am-Be neutron source. Radiat Prot Dosimetry. 2002;98:173-178.

150. Kerns JR, Kry SF, Sahoo N, Followill DS, Ibbott GS. Angular dependence of the nanoDot OSL dosimeter. Med Phys. 2011;38:3955-3962.

151. Saini AS, Zhu TC. Dose rate and SDD dependence of commercially available diode detectors. Med Phys. 2004;31:914-924.

152. Ding GX, Coffey CW. Dosimetric evaluation of the OneDose ${ }^{\mathrm{TM}}$ MOSFET for measuring kilovoltage imaging dose from image-guided radiotherapy procedures. Med Phys. 2010;37:4880-4885.

153. Chiu-Tsao ST, Chan MF. Use of new radiochromic devices for peripheral dose measurement: potential in-vivo dosimetry application. Biomed Imaging Intervention J. 2009;5:e16.11-e16.12.

154. Beddar AS. Plastic scintillation dosimetry and its application to radiotherapy. Radiat Meas. 2006;41:S124-S133.

155. Carrasco P, Jornet N, Jordi O, et al. Characterization of the Exradin W1 scintillator for use in radiotherapy. Med Phys. 2015;42:297-304.

156. Kry SF, Vassiliev ON, Mohan R. Out-of-field photon dose following removal of the flattening filter from a medical accelerator. Phys Med Biol. 2010;55:2155-2166.

157. Kragl G, Baier F, Lutz S, et al. Flattening filter free beams in SBRT and IMRT: dosimetric assessment of peripheral doses. Zeitschrift für Medizinische Physik. 2011;21:91-101.

158. Blais AR, Lederer E, Oliver M, Leszczynski K. Static and rotational step-and-shoot IMRT treatment plans for the prostate: a risk comparison study. Med Phys. 2012;39:1069-1078.

159. Hurkmans CW, Knegjens JL, Oei BS, et al. Management of radiation oncology patients with a pacemaker or ICD: a new comprehensive practical guideline in The Netherlands. Radiation Oncology. 2012;7:198.

160. Zecchin M, Severgnini M, Fiorentino A, et al. Management of patients with cardiac implantable electronic devices (CIED) undergoing radiotherapy: a consensus document from Associazione Italiana Aritmologia e Cardiostimolazione (AIAC), Associazione Italiana Radioterapia Oncologica (AIRO), Associazione Italiana Fisica Medica (AIFM). Int $J$ Cardiol. 2018;255:175-183.

161. Inc MU. Standard Letter, THERAPEUTIC RADIATION [Rev. F, 30APR 2010].

162. Chavez MI. Monitoring patients with implanted cardiac rhythm devices receiving radiation therapy. Oncol Nurs Forum. 2009;36:629-632.

163. Bhandiwad AR, Cummings KW, Crowley M, Woodard PK. Cardiovascular magnetic resonance with an MR compatible pacemaker. $J$ Cardiovasc Magn Reson. 2013;15:18.

164. Harden SP. MRI conditional pacemakers: the start of a new era. $\mathrm{Br} \mathrm{J}$ Radiol. 2011;84:773-774.

165. Wadasadawala T, Pandey A, Agarwal JP, et al. Radiation therapy with implanted cardiac pacemaker devices: a clinical and dosimetric analysis of patients and proposed precautions. Clin Oncol. 2011; 23:79-85.
166. Riley B, Garcia J, Guerrero T. The utilization of a 3-dimensional noncoplanar treatment plan to avoid pacemaker complications. Med Dosim. 2004:29:92-96.

167. de Bie MK, van Rees JB, Thijssen J, et al. Cardiac device infections are associated with a significant mortality risk. Heart Rhythm. 2012;9:494498.

168. Aguilera AL, Volokhina YV, Fisher KL. Radiography of cardiac conduction devices: a comprehensive review. Radiographics. 2011;31:1669-1682.

169. Coolens C, Childs PJ. Calibration of CT Hounsfield units for radiotherapy treatment planning of patients with metallic hip prostheses: the use of the extended CT-scale. Phys Med Biol. 2003;48:1591-1603.

170. Frizzell B. Radiation therapy in oncology patients who have a pacemaker or implantable cardioverter-defibrillator. Commun Oncol. 2009;6:469-471.

171. Reddy K, Cook B, Shaw C, et al. Intact performance of a cochlear implant following radiotherapy in a child with acute lymphoblastic leukemia. Pract Radiat Oncol. 2012;2:233-236.

172. Klenzner T, Knapp F, Rohner F, et al. Influence of ionizing radiation on nucleus 24 cochlear implants. Otol Neurotol. 2005;26:661-667.

173. Klenzner T, Lutterbach J, Aschendorff A, Pedersen P, Stecker M, Laszig R. The effect of large single radiation doses on cochlear implant function: implications for radiosurgery. Eur Arch Otorhinolaryngol. 2004;261:251-255.

174. Ralston A, Stevens G, Mahomudally E, Ibrahim I, Leckie E. Cochlear implants: response to therapeutic irradiation. Int J Radiat Oncol Biol Phys. 1999;44:227-231.

175. Baumann R, Lesinski-Schiedat A, Goldring JE, et al. The influence of ionizing radiation on the CLARION 1.2 cochlear implant during radiation therapy. Am J Otol. 1999;20:50-52.

176. Lauro C, Miften M, Albano E, Wilkinson C, Liu AK. Intact functioning of intrathecal pain pump receiving radiation therapy. J Clin Case Rep. 2012;2:157.

177. Wu H, Wang D. Radiation-induced alarm and failure of an implanted programmable intrathecal pump. Clin J Pain. 2007;23:826-828.

178. Gossman MS, Ketkar A, Liu AK, Olin B. Vagus nerve stimulator stability and interference on radiation oncology x-ray beams. Phys Med Biol. 2012;57:N365-N376.

179. Mitra D, Ghosh K, Gupta P, Jayanti J, Dev A, Sur P. Radiation dose monitoring in a lung cancer patient with a pacemaker-a case report. Indian J Radiol Imaging. 2006;16:875-877.

180. Ng DW, Tondato FJ, Ezzel GA, et al. High incidence of radiotherapyinduced malfunction of permanent pacemakers and ICDs: a single center experience. Europace. 2008;10:I101.

181. Makkar A, Prisciandaro J, Agarwal S, et al. Effect of radiation therapy on permanent pacemaker and implantable cardioverter-defibrillator function. Heart Rhythm. 2012;9:1964-1968.

182. Mihailidis DN. In regard to Gomez et al. Int J Radiat Oncol Biol Phys. 2014;88:753.

183. Mijnheer B, Beddar S, Izewska J, Reft C. In vivo dosimetry in external beam radiotherapy. Med Phys. 2013;40(7):070903. 